IMA Journal of Numerical Analysis (2016) Page 1 of 29

doi:10.1093/imanum/drnxxx

\title{
A Convergent Adaptive Finite Element Method for Electrical Impedance Tomography
}

\author{
BANGTI JIN $\dagger$ \\ Department of Computer Science, University College London \\ Gower Street, London WCIE 6BT, UK \\ YIFENG XU‡ \\ Department of Mathematics, Scientific Computing Key Laboratory of Shanghai Universities \\ and E-Institute for Computational Science of Shanghai Universities, Shanghai Normal \\ University, Shanghai 200234, P.R. China \\ JuN Zou§ \\ Department of Mathematics, The Chinese University of Hong Kong \\ Shatin, New Territories, Hong Kong, P.R. China
}

[Received on November 11, 2015; revised on April 24, 2016]

\begin{abstract}
In this work we develop and analyze an adaptive finite element method for efficiently solving electrical impedance tomography - a severely ill-posed nonlinear inverse problem of recovering the conductivity from boundary voltage measurements. The reconstruction technique is based on Tikhonov regularization with a Sobolev smoothness penalty and discretizing the forward model using continuous piecewise linear finite elements. We derive an adaptive finite element algorithm with an a posteriori error estimator involving the concerned state and adjoint variables and the recovered conductivity. The convergence of the algorithm is established, in the sense that the sequence of discrete solutions contains a convergent subsequence to a solution of the optimality system for the continuous formulation. Numerical results are presented to verify the convergence and efficiency of the algorithm.
\end{abstract}

Keywords: electrical impedance tomography, a posteriori error estimator, adaptive finite element method, convergence analysis.

\section{Introduction}

Electrical impedance tomography (EIT) is a diffusive imaging modality for probing internal structures of the concerned object, by recovering its electrical conductivity/permittivity distribution from voltage measurements on the boundary. One typical experimental setup is as follows. One first attaches a set of metallic electrodes to the surface of the object, then injects an input current into the object through these electrodes, which induces an electromagnetic field inside the object. Last, one measures the induced electric voltages on the electrodes. The procedure is usually repeated several times with different input currents in order to yield sufficient information about the sought-for conductivity distribution. In many applications, the physical process can be most accurately described by the complete electrode model (CEM) (Cheng et al. (1989), Somersalo et al. (1992)). The imaging modality has attracted considerable interest in medical imaging, geophysical prospecting, nondestructive evaluation and pneumatic oil pipeline conveying etc.

\footnotetext{
${ }^{\dagger}$ Email: bangti.jin@gmail.com, b.jin@ucl.ac.uk

ॠCorresponding author. Email: yfxu@shnu.edu.cn, mayfxu@gmail.com

§Email: zou@math.cuhk.edu.hk
} 
A number of reconstruction algorithms have been proposed for the EIT inverse problem; see, e.g., Lechleiter et al. (2008), Adler et al. (2011), Jin et al. (2012), Jin \& Maass (2012a), Lechleiter \& Rieder (2006), Winkler \& Rieder (2014), Knudsen et al. (2009), Harrach \& Ullrich (2013), Gehre \& Jin (2014), Chow et al. (2014), Dunlop \& Stuart (2015) for an incomplete list. One prominent idea underlying existing imaging algorithms is regularization, especially Tikhonov regularization with a smoothness or sparsity type penalty, and they have demonstrated encouraging results with experimental data. In practice, they are customarily implemented using the continuous piecewise linear finite element method (FEM), due to its flexibility in handling variable coefficients and general geometry. Despite its popularity, it was only rigorously justified recently in Gehre et al. (2014) for the CEM on either polygonal or smooth convex domains.

The accuracy of the CEM relies crucially on the use of nonstandard boundary conditions for capturing important characteristics of the physical experiment, notably contact impedance effect. As a consequence, around the boundary of the electrodes, the boundary condition changes from the Neumann to Robin type, which induces weak singularity of the forward solution around the interface (Grisvard (1985)). The lowregularity of the sought-for conductivity field, as enforced by Sobolev smoothness penalty, will possibly also induce weak solution singularities. With a quasi-uniform triangulation of the domain, the solution singularities are not effectively resolved and the errors around electrode edges and discontinuity interfaces are dominant, which can potentially compromise the reconstruction accuracy greatly, if done inadvertently. This naturally motivates the use of an adaptive strategy to achieve the desired accuracy with reduced computational complexity. In this work, we shall develop a novel adaptive finite element method (AFEM) for the EIT inverse problem and analyze its convergence.

Generally the AFEM generates a sequence of nested triangulations and discrete solutions by the following successive loop:

$$
\text { SOLVE } \rightarrow \text { ESTIMATE } \rightarrow \text { MARK } \rightarrow \text { REFINE. }
$$

The key ingredient in the procedure is the module ESTIMATE, which consists of computing a posteriori error estimators, i.e., computable quantities from the discrete solution, the local mesh size and other given data. This has been thoroughly studied for forward problems; see, e.g., Ainsworth \& Oden (2000), Verfürth (1996). Over the past few decades, there are also many important works on the a posteriori error analysis of PDE-constrained optimal control problems; see Hintermüller \& Hoppe (2010), Hintermüller et al. (2008), Li et al. (2002), Liu \& Yan (2001), Becker \& Mao (2011) for a very incomplete list. In particular, Becker \& Mao (2011) showed the quasi-optimality of the AFEM for an optimal control problem with control constraints. However, the behavior of inverse problems such as EIT is quite different from that of optimal control problems due to the ill-posed nature, the presence of the data noise and high-degree nonlinearity.

The adaptive idea, including the AFEM, has started to attract some attention in the context of inverse problems in recent years. In Becker \& Vexler (2004), Beilina \& Clason (2006), Beilina \& Johnson (2005), the AFEM using a dual weighted residual framework was studied for parameter identification problems, and high order terms in relevant Lagrangian functionals were ignored. Feng et al. (2008) proposed a residualbased estimator for state, costate (adjoint) and parameter by assuming convexity of the cost functional and high regularity on the parameter. Li et al. (2011) derived rigorous a posteriori error estimators for reconstructing the distributed flux under a practical regularity assumption, in the sense that like for forward problems, the errors of the state variable, the adjoint variable and the flux are bounded from above and below by multiples of the estimators. In a series of interesting works (Beilina \& Klibanov (2010a,b); Beilina et al. (2010)), Beilina et al adopted the AFEM for hyperbolic coefficient inverse problems. Griesbaum et al. (2008) and Kaltenbacher et al. (2014) described and analyzed adaptive strategies for choosing the regularization parameter in Tikhonov regularization and iterative regularization techniques, e.g., Gauss-Newton methods. Unlike the AFEM for forward problems, for which the convergence and computational complexity have been systematically studied (see the survey papers Carstensen et al. (2014), Nochetto et al. (2009)), the 
theoretical analysis of the AFEM for inverse problems is still in its infancy. Recently, Xu \& Zou (2015b,a) established the convergence of the AFEM for recovering the flux and the Robin coefficient. We remark that the convergence rate and optimality of the AFEM in the context of nonlinear inverse problems are completely open, due to inherent nonconvexity of the functional, and lack of precise regularity results of the minimizers to the nonlinear optimization problem. Nonetheless, our convergence result in Theorem 4.10 provides some theoretical justifications of the AFEM for the EIT inverse problem.

In this paper, we develop a novel AFEM for the EIT based on Tikhonov regularization with a $H^{1}(\Omega)$ seminorm penalty and analyze its convergence. The AFEM is of the standard form (1.1): it does not require the interior node property in the module REFINE, and hence it is easy to implement. The derivation of a posteriori error estimators is constructive: it lends itself to a route for convergence analysis. The analysis relies on a limiting output least-squares problem defined on the closure of adaptively generated finite element spaces, and it consists of the following two steps. First, the sequence of discrete minimizers is shown in Section 4.1 to contain a subsequence converging to a solution of the limiting problem, and then the limiting minimizer and related state and adjoint variables are proved in Section 4.2 to satisfy the necessary optimality system of the continuous Tikhonov functional.

This work is a continuation of our prior work Gehre et al. (2014) on the FEM analysis of EIT, but differs from the latter considerably in several aspects. The major effort of Gehre et al. (2014) was to justify the convergence of the quasiuniform FEM approximation of the Tikhonov formulation of the EIT, and no a posteriori error estimator and adaptive method were studied, which is the main goal of the present work. The convergence analysis in Gehre et al. (2014) relies crucially on the $W^{1, q}(\Omega)(q>2)$ regularity of the forward solution and the density of FE spaces $V_{h}$ in $H^{1}(\Omega)$. The density does not hold generally for adaptively generated FE spaces. Hence, the analysis in Gehre et al. (2014) does not carry over to the AFEM directly. In this work, we shall adopt a strategy developed in Xu \& Zou (2015a) for recovering the Robin coefficient from the Cauchy data to overcome these technical difficulties. Nonetheless, there are major differences in the analysis due to higher degree of nonlinearity of the EIT problem. In Xu \& Zou (2015a), the continuity of the parameter-to-state map from $L^{2}\left(\Gamma_{i}\right)$ to $L^{2}\left(\Gamma_{c}\right)$ plays a crucial role. For the EIT, only the $H^{1}(\Omega)$ weak continuity of the forward map holds (cf. Lemma 4.3), and we shall exploit the pointwise convergence of discrete minimizers and Lebesgue's dominated convergence theorem. This allows us to establish the $H^{1}(\Omega)$ convergence of discrete state variables (cf. Theorem 4.4), and thus enables us to verify that the limiting solution also satisfies the optimality system of the continuous functional (Lemmas 4.8 and 4.9).

The rest of this paper is organized as follows. In Section 2, we describe the CEM, regularized leastsquares formulation and its necessary optimality system. The finite element discretization is described, and an adaptive FEM algorithm for the EIT is proposed in Section 3, where a heuristic yet constructive derivation is also provided. The convergence analysis of the adaptive algorithm is given in Section 4. Some numerical results are given in Section 5 to illustrate its convergence and efficiency. We conclude the section with some notation. We shall use the standard notation for Sobolev spaces, following Evans \& Gariepy (1992). Further, we use $\langle\cdot, \cdot\rangle$ and $(\cdot, \cdot)$ to denote the inner product on the Euclidean space and $\left(L^{2}(\Omega)\right)^{d}$, respectively, by $\|\cdot\|$ the Euclidean norm, and occasionally abuse $\langle\cdot, \cdot\rangle$ for the duality pairing between the space $\mathbb{H}$ and its dual space. Throughout, the notation $c$ denotes a generic constant, which may differ at each occurrence, but is always independent of the mesh size and other quantities of interest.

\section{Preliminaries}

We shall recall in this section the mathematical model for the EIT problem and describe the reconstruction technique based on Tikhonov regularization and its necessary optimality system. 


\subsection{Complete electrode model}

Let $\Omega$ be an open bounded domain in $\mathbb{R}^{d}(d=2,3)$ with a polyhedral boundary $\Gamma$. We denote the set of electrodes by $\left\{e_{l}\right\}_{l=1}^{L}$, which are line segments/planar surfaces on $\Gamma$ and disjoint from each other, i.e., $\bar{e}_{i} \cap \bar{e}_{k}=\emptyset$ if $i \neq k$. The applied current on the $l$ th electrode $e_{l}$ is denoted by $I_{l}$, and the current vector $I=\left(I_{1}, \ldots, I_{L}\right)^{\mathrm{t}}$ satisfies $\sum_{l=1}^{L} I_{l}=0$ by the law of charge conservation. Let the space $\mathbb{R}_{\diamond}^{L}$ be the subspace of the vector space $\mathbb{R}^{L}$ with zero mean. Then we have $I \in \mathbb{R}_{\diamond}^{L}$. The electrode voltage $U=\left(U_{1}, \ldots, U_{L}\right)^{\mathrm{t}}$ is also normalized such that $U \in \mathbb{R}_{\diamond}^{L}$. Then the CEM reads: given the conductivity $\sigma$, positive contact impedances $\left\{z_{l}\right\}_{l=1}^{L}$ and input current $I \in \mathbb{R}_{\diamond}^{L}$, find the potential $u \in H^{1}(\Omega)$ and electrode voltage $U \in \mathbb{R}_{\diamond}^{L}$ such that

$$
\begin{cases}-\nabla \cdot(\sigma \nabla u)=0 & \text { in } \Omega, \\ u+z_{l} \sigma \frac{\partial u}{\partial n}=U_{l} & \text { on } e_{l}, l=1,2, \ldots, L, \\ \int_{e_{l}} \sigma \frac{\partial u}{\partial n} d s=I_{l} & \text { for } l=1,2, \ldots, L, \\ \sigma \frac{\partial u}{\partial n}=0 & \text { on } \Gamma \backslash \cup_{l=1}^{L} e_{l} .\end{cases}
$$

The physical motivation behind the model (2.1) is as follows. The governing equation is derived under a quasi-static assumption on the electromagnetic process. The second line describes the contact impedance effect: When injecting electrical currents into the object, a highly resistive thin layer forms at the electrodeelectrolyte interface, which causes potential drops across the electrode-electrolyte interface. The potential drop is described by Ohm's law, with proportionality factors $\left\{z_{l}\right\}_{l=1}^{L}$. It also takes into account the fact that metallic electrodes are perfect conductors, and hence the voltage $U_{l}$ is constant on each electrode. The third line reflects the fact that the current $I_{l}$ injected through the electrode $e_{l}$ is completely confined to $e_{l}$ itself. The nonstandard boundary conditions is essential for the model (2.1) to reproduce experimental data within the measurement precision; see Cheng et al. (1989) and Somersalo et al. (1992).

Due to physical constraint, the conductivity distribution $\sigma$ is naturally bounded both from below and above by positive constants. Hence we introduce the following admissible set $\mathscr{A}$ : for some $\lambda \in(0,1)$, let

$$
\mathscr{A}=\left\{\lambda \in H^{1}(\Omega): \lambda \leqslant \sigma(x) \leqslant \lambda^{-1} \text { a.e. } x \in \Omega\right\} .
$$

The set $\mathscr{A}$ is endowed with the $H^{1}(\Omega)$-norm, in view of the $H^{1}(\Omega)$-seminorm regularization, cf. (2.3) below. Further, we denote by $\mathbb{H}$ the product space $H^{1}(\Omega) \otimes \mathbb{R}_{\diamond}^{L}$ with its norm defined by

$$
\|(u, U)\|_{\mathbb{H}}^{2}=\|u\|_{H^{1}(\Omega)}^{2}+\|U\|^{2} .
$$

A convenient equivalent norm on the space $\mathbb{H}$ is given below.

LEMMA 2.1 On the space $\mathbb{H}$, the norm $\|\cdot\|_{\mathbb{H}}$ is equivalent to the norm $\|\cdot\|_{\mathbb{H}, *}$ defined by

$$
\|(u, U)\|_{\mathbb{H}, *}^{2}=\|\nabla u\|_{L^{2}(\Omega)}^{2}+\sum_{l=1}^{L}\left\|u-U_{l}\right\|_{L^{2}\left(e_{l}\right)}^{2} .
$$

Proof. The lemma is a folklore result in the EIT community, and we provide a proof only for completeness. It is easy to verify that $\|(u, U)\|_{\mathbb{H}, *}$ indeed defines a proper norm. It suffices to show the following two inequalities: there exist $c_{1}, c_{2}>0$ such that

$$
c_{1}\|(u, U)\|_{\mathbb{H}} \leqslant\|(u, U)\|_{\mathbb{H}, *} \leqslant c_{2}\|(u, U)\|_{\mathbb{H}} \cdot
$$

The second inequality follows from the Cauchy-Schwarz inequality and trace theorem. We show the first inequality by contradiction. Assume the contrary. Then there exists a sequence $\left\{\left(u^{n}, U^{n}\right)\right\} \subset \mathbb{H}$ such that 
$\left\|\left(u^{n}, U^{n}\right)\right\|_{\mathbb{H}}=1$ and $\left\|\left(u^{n}, U^{n}\right)\right\|_{\mathbb{H}, *}<n^{-1}$. Then there exists a convergent subsequence, also denoted by $\left\{u^{n}\right\}$, to some $u \in H^{1}(\Omega)$ weakly in $H^{1}(\Omega)$. By the compact embedding of $H^{1}(\Omega)$ into $L^{2}(\Omega)$, the sequence $\left\{u^{n}\right\}$ converges to $u$ in $L^{2}(\Omega)$. Further, by construction, $\left\|\nabla u^{n}\right\|_{L^{2}(\Omega)} \leqslant n^{-1}$. Thus $\left\{u^{n}\right\}$ converges to $u$ in $H^{1}(\Omega)$, and $u=c$ in the domain $\Omega$ for some $c \in \mathbb{R}$. By trace theorem and Sobolev embedding theorem, $\left\{u^{n}\right\}$ converges to $u$ in $L^{2}(\Gamma)$. Since $\left\|u^{n}-U_{l}^{n}\right\|_{L^{2}\left(e_{l}\right)}<n^{-1},\left\{U_{l}^{n}\right\}$ converges to the trace of $u$ on $e_{l}$ for each $l=1,2, \ldots, L$, i.e., the limit $U=c(1, \ldots, 1)^{T}$. Now the condition $U \in \mathbb{R}_{\diamond}^{L}$ implies $U=0, c=0$ and $u \equiv 0$. Consequently, $u^{n} \rightarrow 0$ in $H^{1}(\Omega)$ and $U^{n} \rightarrow 0$ in $\mathbb{R}^{L}$, which contradicts the assumption $\left\|\left(u^{n}, U^{n}\right)\right\|_{\mathbb{H}}=1$.

The weak formulation of the model $(2.1)$ reads: find $(u, U) \in \mathbb{H}$ such that

$$
a(\sigma,(u, U),(v, V))=\langle I, V\rangle \quad \forall(v, V) \in \mathbb{H},
$$

where the trilinear form $a(\sigma,(u, U),(v, V))$ on $\mathscr{A} \times \mathbb{H} \times \mathbb{H}$ is defined by

$$
a(\sigma,(u, U),(v, V))=(\sigma \nabla u, \nabla v)+\sum_{l=1}^{L} z_{l}^{-1}\left(u-U_{l}, v-V_{l}\right)_{L^{2}\left(e_{l}\right)},
$$

where $(\cdot, \cdot)_{L^{2}\left(e_{l}\right)}$ denotes the $L^{2}\left(e_{l}\right)$ inner product. By Lemma 2.1, for any $\sigma \in \mathscr{A}$, the bilinear form $a(\sigma, \cdot, \cdot)$ is continuous and coercive on the space $\mathbb{H}$. Hence, by Lax-Milgram theorem, for any fixed $\sigma \in \mathscr{A}$ and given contact impedances $\left\{z_{l}\right\}_{l=1}^{L}$ and current $I \in \Sigma_{\diamond}^{L}$, there exists a unique solution $(u, U) \equiv(u(\sigma), U(\sigma)) \in \mathbb{H}$ to (2.2), and it depends continuously on the input current pattern $I$. Since $\sigma \in \mathscr{A}$, one can deduce that $u \in W^{1, q}(\Omega)$ for some $q>2$ (Jin \& Maass (2012a)). See also Jin \& Maass (2012a), Gehre et al. (2014) and Dunlop \& Stuart (2015) for various continuity results of $(u, U)$ with respect to the conductivity $\sigma$.

REMARK 2.2 Alternatively, one can formulate a proper variational formulation of the CEM (2.1) on the quotient space $\mathbb{H}=\left(H^{1}(\Omega) \times \mathbb{R}^{L}\right) / \mathbb{R}$, with the norm defined by

$$
\|(u, U)\|_{\dot{H}}=\inf _{c \in \mathbb{R}}\left(\|u-c\|_{H^{1}(\Omega)}^{2}+\|U-c\|^{2}\right)^{1 / 2} .
$$

Then the bilinear form $a(\sigma, \cdot, \cdot)$ is continuous and coercive on the space $\mathbb{H}$; see Somersalo et al. (1992) for details. It differs from the preceding one in the grounding condition: in the choice $\mathbb{H}$, the grounding is enforced by the zero mean condition $U \in \mathbb{R}_{\diamond}^{L}$.

\subsection{Tikhonov regularization}

The inverse problem is to reconstruct the conductivity $\sigma$ from noisy measurements $U^{\delta}$ of the exact electrode voltage $U\left(\sigma^{\dagger}\right)$, corresponding to one or multiple input currents, with a noise level $\delta$ :

$$
\left\|U^{\delta}-U\left(\sigma^{\dagger}\right)\right\| \leqslant \delta
$$

It is severely ill-posed in the sense that small errors in the data can lead to very large deviations in the reconstructions. Hence, some sort of regularization is beneficial, and it is incorporated into imaging algorithms, either implicitly or explicitly, in order to yield physically meaningful images. One prominent idea behind many existing imaging algorithms is Tikhonov regularization, which minimizes the following functional

$$
\min _{\sigma \in \mathscr{A}}\left\{J(\sigma)=\frac{1}{2}\left\|U(\sigma)-U^{\delta}\right\|^{2}+\frac{\alpha}{2}\|\nabla \sigma\|_{L^{2}(\Omega)}^{2}\right\}
$$

and then takes the minimizer as an approximation to the true conductivity $\sigma^{\dagger}$. The first term in the functional $J$ integrates the information in the data $U^{\delta}$. For notational simplicity, we consider only one dataset in the 
discussion, and the adaptation to multiple datasets is straightforward. The second term imposes a priori regularity assumption (Sobolev smoothness) on the expected conductivity $\sigma$. The scalar $\alpha>0$ is known as a regularization parameter, and controls the tradeoff between the two terms (Ito \& Jin (2015)). Problem (2.3) has at least one minimizer, and it depends continuously on the data perturbation (Jin \& Maass (2012a)). The convergence of the Tikhonov minimizer to $\sigma^{\dagger}$ as the noise level $\delta$ tends to zero was shown in Jin \& Maass (2012a), if the true conductivity $\sigma^{\dagger} \in H^{1}(\Omega)$, and also a convergence rate $O\left(\delta^{1 / 2}\right)$ was given under suitable source condition as $\delta \rightarrow 0$, both under a proper choice of regularization parameter $\alpha$.

Following the standard adjoint technique (see, e.g., Ito \& Kunisch (2008)), we introduce the following adjoint problem for (2.2): find $(p, P) \equiv(p(\sigma), P(\sigma)) \in \mathbb{H}$ such that

$$
a(\sigma,(p, P),(v, V))=\left\langle U(\sigma)-U^{\delta}, V\right\rangle \quad \forall(v, V) \in \mathbb{H} .
$$

Then it can be verified that the Gâteaux derivative of $J(\sigma)$ at $\sigma \in \mathscr{A}$ in the direction $\mu$ is given by

$$
J^{\prime}(\sigma)[\mu]=(\alpha \nabla \sigma, \nabla \mu)-(\mu \nabla u(\sigma), \nabla p(\sigma)) .
$$

Then the minimizer $\sigma^{*}$ to problem (2.3) and the respective forward solution $\left(u^{*}, U^{*}\right)$ and the adjoint solution $\left(p^{*}, P^{*}\right)$ satisfies the following necessary optimality system:

$$
\begin{aligned}
& a\left(\sigma^{*},\left(u^{*}, U^{*}\right),(v, V)\right)=\langle I, V\rangle \quad \forall(v, V) \in \mathbb{H}, \\
& a\left(\sigma^{*},\left(p^{*}, P^{*}\right),(v, V)\right)=\left\langle U^{*}-U^{\delta}, V\right\rangle \quad \forall(v, V) \in \mathbb{H}, \\
& \alpha\left(\nabla \sigma^{*}, \nabla\left(\mu-\sigma^{*}\right)\right)-\left(\left(\mu-\sigma^{*}\right) \nabla u^{*}, \nabla p^{*}\right) \geqslant 0 \quad \forall \mu \in \mathscr{A},
\end{aligned}
$$

where the variational inequality at the last line corresponds to the box constraint in the admissible set $\mathscr{A}$.

\section{Adaptive finite element method}

Now we describe the finite element method (FEM) for discretizing problem (2.3), derive the a posteriori error estimator and develop a novel adaptive algorithm, which uses a general marking strategy and thus is easy to implement. The convergence analysis of the algorithm will be presented in Section 4.

\subsection{Finite element discretization}

To discretize the problem, we first triangulate the domain $\Omega$. Let $\mathscr{T}$ be a shape regular triangulation of the polyhedral domain $\bar{\Omega}$ consisting of closed simplicial elements, with a local mesh size $h_{T}:=|T|^{1 / d}$ for each element $T \in \mathscr{T}$, which is assumed to intersect at most one electrode surface $e_{l}$. On the triangulation $\mathscr{T}$, we define a continuous piecewise linear finite element space

$$
V_{\mathscr{T}}=\left\{v \in C(\bar{\Omega}):\left.v\right|_{T} \in P_{1}(T) \forall T \in \mathscr{T}\right\},
$$

where the space $P_{1}(T)$ consists of all linear functions on the element $T$. The space $V_{\mathscr{T}}$ is also used for approximating the potential $u$ and the conductivity $\sigma$. The use of piecewise linear finite elements is popular since the problem data, e.g., boundary conditions, have only limited regularity.

Now we can describe the FEM approximation. First, we approximate the forward map $(u(\sigma), U(\sigma)) \in \mathbb{H}$ by $\left(u_{\mathscr{T}}, U_{\mathscr{T}}\right) \equiv\left(u_{\mathscr{T}}\left(\sigma_{\mathscr{T}}\right), U_{\mathscr{T}}\left(\sigma_{\mathscr{T}}\right)\right) \in \mathbb{H}_{\mathscr{T}} \equiv V_{\mathscr{T}} \otimes \mathbb{R}_{\diamond}^{L}$ defined by

$$
a\left(\sigma_{\mathscr{T}},\left(u_{\mathscr{T}}, U_{\mathscr{T}}\right),\left(v_{\mathscr{T}}, V\right)\right)=\langle I, V\rangle \quad\left(v_{\mathscr{T}}, V\right) \in \mathbb{H}_{\mathscr{T}},
$$


where the (discretized) conductivity $\sigma_{\mathscr{T}}$ lies in the discrete admissible set

$$
\mathscr{A}_{\mathscr{T}}=\left\{\sigma_{\mathscr{T}} \in V_{\mathscr{T}}: \lambda \leqslant \sigma_{\mathscr{T}} \leqslant \lambda^{-1} \text { a.e. } \Omega\right\}=\mathscr{A} \cap V_{\mathscr{T}} .
$$

Then the discrete optimization problem reads

$$
\min _{\sigma_{\mathscr{T}} \in \mathscr{A}_{\mathscr{T}}}\left\{J_{\mathscr{T}}\left(\sigma_{\mathscr{T}}\right)=\frac{1}{2}\left\|U_{\mathscr{T}}\left(\sigma_{\mathscr{T}}\right)-U^{\delta}\right\|^{2}+\frac{\alpha}{2}\left\|\nabla \sigma_{\mathscr{T}}\right\|_{L^{2}(\Omega)}^{2}\right\}
$$

Due to the compactness of the finite-dimensional space $\mathscr{A}_{\mathscr{T}}$, it is easy to see that there exists at least one minimizer $\sigma_{\mathscr{T}}^{*}$ to problem (3.1)-(3.2) (see, e.g., Gehre et al. (2014)). The minimizer $\sigma_{\mathscr{T}}^{*}$ and the related forward solution $\left(u_{\mathscr{T}}^{*}, U_{\mathscr{T}}^{*}\right) \equiv\left(u_{\mathscr{T}}^{*}\left(\sigma_{\mathscr{T}}^{*}\right), U_{\mathscr{T}}^{*}\left(\sigma_{\mathscr{T}}^{*}\right)\right) \in \mathbb{H}_{\mathscr{T}}$ and adjoint solution $\left(p_{\mathscr{T}}^{*}, P_{\mathscr{T}}^{*}\right) \equiv\left(p_{\mathscr{T}}^{*}\left(\sigma_{\mathscr{T}}^{*}\right), P_{\mathscr{T}}^{*}\left(\sigma_{\mathscr{T}}^{*}\right)\right) \in$ $\mathbb{H}_{\mathscr{T}}$ satisfies the following necessary optimality system

$$
\begin{aligned}
& a\left(\sigma_{\mathscr{T}}^{*},\left(u_{\mathscr{T}}^{*}, U_{\mathscr{T}}^{*}\right),\left(v_{\mathscr{T}}, V\right)\right)=\langle I, V\rangle \quad \forall\left(v_{\mathscr{T}}, V\right) \in \mathbb{H}_{\mathscr{T}}, \\
& a\left(\sigma_{\mathscr{T}}^{*},\left(p_{\mathscr{T}}^{*}, P_{\mathscr{T}}^{*}\right),\left(v_{\mathscr{T}}, V\right)\right)=\left\langle U_{\mathscr{T}}^{*}-U^{\delta}, V\right\rangle \quad \forall\left(v_{\mathscr{T}}, V\right) \in \mathbb{H}_{\mathscr{T}}, \\
& \alpha\left(\nabla \sigma_{\mathscr{T}}^{*}, \nabla\left(\mu_{\mathscr{T}}-\sigma_{\mathscr{T}}^{*}\right)\right)-\left(\left(\mu_{\mathscr{T}}-\sigma_{\mathscr{T}}^{*}\right) \nabla u_{\mathscr{T}}^{*}, \nabla p_{\mathscr{T}}^{*}\right) \geqslant 0 \quad \forall \mu_{\mathscr{T}} \in \mathscr{A}_{\mathscr{T}},
\end{aligned}
$$

which is the discrete analogue of (2.5). Like in the continuous case, it is straightforward to verify that the discrete solutions $\left(u_{\mathscr{T}}^{*}, U_{\mathscr{T}}^{*}\right)$ and $\left(p_{\mathscr{T}}^{*}, P_{\mathscr{T}}^{*}\right)$ depend continuously on the input current pattern $I$, i.e.,

$$
\left\|\left(u_{\mathscr{T}}^{*}, U_{\mathscr{T}}^{*}\right)\right\|_{\mathbb{H}, *}+\left\|\left(p_{\mathscr{T}}^{*}, U_{\mathscr{T}}^{*}\right)\right\|_{\mathbb{H}, *} \leqslant c\left(\|I\|+\left\|U^{\delta}\right\|\right),
$$

where the constant $c$ can be made independent of $\alpha$.

\subsection{Adaptive algorithm}

Now we can present a novel AFEM for problem (2.2)-(2.3). First we introduce some notation. Let $\mathbb{T}$ be the set of all possible conforming triangulations of the domain $\bar{\Omega}$ obtained from some shape-regular initial mesh $\mathscr{T}_{0}$ by the successive use of bisection. We call $\mathscr{T}^{\prime} \in \mathbb{T}$ a refinement of $\mathscr{T} \in \mathbb{T}$ if $\mathscr{T}^{\prime}$ can be obtained from $\mathscr{T}$ by a finite number of bisections. The collection of all faces (respectively all interior faces) in $\mathscr{T} \in \mathbb{T}$ is denoted by $\mathscr{F}_{\mathscr{T}}$ (respectively $\mathscr{F}_{\mathscr{T}}^{i}$ ) and its restriction on the electrode $\bar{e}_{l}$ and $\Gamma \backslash \cup_{l=1}^{L} e_{l}$ by $\mathscr{F}_{\mathscr{T}}^{l}$ and $\mathscr{F}_{\mathscr{T}}^{c}$, respectively. The scalar $h_{F}:=|F|^{1 /(d-1)}$ denotes the diameter of a face $F \in \mathscr{F}_{\mathscr{T}}$, which is associated with a fixed normal unit vector $\boldsymbol{n}_{F}$ in $\bar{\Omega}$ with $\boldsymbol{n}_{F}=\boldsymbol{n}$ on the boundary $\Gamma$. Further, we denote by $D_{T}$ (respectively $D_{F}$ ) the union of all elements in $\mathscr{T}$ with non-empty intersection with an element $T \in \mathscr{T}$ (respectively $F \in \mathscr{F}_{\mathscr{T}}$ ).

REMARK 3.1 Our convergence analysis covers any bisection method that ensures that the family $\mathbb{T}$ is uniformly shape regular during the refinement process, i.e., shape regularity of any $\mathscr{T} \in \mathbb{T}$ is uniformly bounded by a constant depending only on the initial mesh $\mathscr{T}_{0}$ (Nochetto et al., 2009, Lemma 4.1), and thus all constants only depend on the initial mesh $\mathscr{T}_{0}$ and given data but not on any subsequent mesh. Such bisection methods include in particular newest vertex bisection in two dimensions (Mitchell (1989)) and the bisection of Kossaczký (1995) in three dimensions. Note that no interior node property is enforced between two consecutive refinements by bisection in our AFEM.

For the solution $\left(\sigma_{\mathscr{T}}^{*}, u_{\mathscr{T}}^{*}, U_{\mathscr{T}}^{*}, p_{\mathscr{T}}^{*}, P_{\mathscr{T}}^{*}\right)$ to problem (3.3), we define two element residuals for each element $T \in \mathscr{T}$ and two face residuals for each face $F \in \mathscr{F}_{\mathscr{T}}$ by

$$
\begin{aligned}
& R_{T, 1}\left(\sigma_{\mathscr{T}}^{*}, u_{\mathscr{T}}^{*}\right)=\nabla \cdot\left(\sigma_{\mathscr{T}}^{*} \nabla u_{\mathscr{T}}^{*}\right), \\
& R_{T, 2}\left(u_{\mathscr{T}}^{*}, p_{\mathscr{T}}^{*}\right)=\nabla u_{\mathscr{T}}^{*} \cdot \nabla p_{\mathscr{T}}^{*},
\end{aligned}
$$




$$
\begin{aligned}
J_{F, 1}\left(\sigma_{\mathscr{T}}^{*}, u_{\mathscr{T}}^{*}, U_{\mathscr{T}}^{*}\right)= \begin{cases}{\left[\sigma_{\mathscr{T}}^{*} \nabla u_{\mathscr{T}}^{*} \cdot \boldsymbol{n}_{F}\right]} & \text { for } F \in \mathscr{F}_{\mathscr{T}}^{i}, \\
\sigma_{\mathscr{T}}^{*} \nabla u_{\mathscr{T}}^{*} \cdot \boldsymbol{n}+\left(u_{\mathscr{T}}^{*}-U_{\mathscr{T}, l}^{*}\right) / z_{l} & \text { for } F \in \mathscr{F}_{\mathscr{T}}^{l}, \\
\sigma_{\mathscr{T}}^{*} \nabla u_{\mathscr{T}}^{*} \cdot \boldsymbol{n} & \text { for } F \in \mathscr{F}_{\mathscr{T}}^{c},\end{cases} \\
J_{F, 2}\left(\sigma_{\mathscr{T}}^{*}\right)= \begin{cases}{\left[\alpha \nabla \sigma_{\mathscr{T}}^{*} \cdot \boldsymbol{n}_{F}\right]} & \text { for } F \in \mathscr{F}_{\mathscr{T}}^{i}, \\
\alpha \nabla \sigma_{\mathscr{T}}^{*} \cdot \boldsymbol{n} & \text { for } F \in \mathscr{F}_{\mathscr{T}}^{l} \cup \mathscr{F}_{\mathscr{T}}^{c},\end{cases}
\end{aligned}
$$

where [.] denotes the jumps across interior faces $F$. Then for any collection of elements $\mathscr{M}_{\mathscr{T}} \subseteq \mathscr{T}$, we introduce the following error estimator

$$
\begin{aligned}
\eta_{\mathscr{T}}^{2}\left(\sigma_{\mathscr{T}}^{*}, u_{\mathscr{T}}^{*}, U_{\mathscr{T}}^{*}, p_{\mathscr{T}}^{*}, P_{\mathscr{T}}^{*}, \mathscr{M}_{\mathscr{T}}\right):=\sum_{T \in \mathscr{M}_{\mathscr{T}}} \eta_{\mathscr{T}}^{2}\left(\sigma_{\mathscr{T}}^{*}, u_{\mathscr{T}}^{*}, U_{\mathscr{T}}^{*}, p_{\mathscr{T}}^{*}, P_{\mathscr{T}}^{*}, T\right) \\
\quad:=\sum_{T \in \mathscr{M}_{\mathscr{T}}} \eta_{\mathscr{T}, 1}^{2}\left(\sigma_{\mathscr{T}}^{*}, u_{\mathscr{T}}^{*}, U_{\mathscr{T}}^{*}, T\right)+\eta_{\mathscr{T}, 2}^{2}\left(\sigma_{\mathscr{T}}^{*}, p_{\mathscr{T}}^{*}, P_{\mathscr{T}}^{*}, T\right)+\eta_{\mathscr{T}, 3}^{2}\left(\sigma_{\mathscr{T}}^{*}, u_{\mathscr{T}}^{*}, p_{\mathscr{T}}^{*}, T\right),
\end{aligned}
$$

where the three components $\eta_{\mathscr{T}, i}^{2}, i=1,2,3$, are defined by

$$
\begin{aligned}
& \eta_{\mathscr{T}, 1}^{2}\left(\sigma_{\mathscr{T}}^{*}, u_{\mathscr{T}}^{*}, U_{\mathscr{T}}^{*}, T\right):=h_{T}^{2}\left\|R_{T, 1}\left(\sigma_{\mathscr{T}}^{*}, u_{\mathscr{T}}^{*}\right)\right\|_{L^{2}(T)}^{2}+\sum_{F \subset \partial T} h_{F}\left\|J_{F, 1}\left(\sigma_{\mathscr{T}}^{*}, u_{\mathscr{T}}^{*}, U_{\mathscr{T}}^{*}\right)\right\|_{L^{2}(F)}^{2}, \\
& \eta_{\mathscr{T}, 2}^{2}\left(\sigma_{\mathscr{T}}^{*}, p_{\mathscr{T}}^{*}, P_{\mathscr{T}}^{*}, T\right):=h_{T}^{2}\left\|R_{T, 1}\left(\sigma_{\mathscr{T}}^{*}, p_{\mathscr{T}}^{*}\right)\right\|_{L^{2}(T)}^{2}+\sum_{F \subset \partial T} h_{F}\left\|J_{F, 1}\left(\sigma_{\mathscr{T}}^{*}, p_{\mathscr{T}}^{*}, P_{\mathscr{T}}^{*}\right)\right\|_{L^{2}(F)}^{2}, \\
& \eta_{\mathscr{T}, 3}^{2}\left(\sigma_{\mathscr{T}}^{*}, u_{\mathscr{T}}^{*}, p_{\mathscr{T}}^{*}, T\right):=h_{T}^{4}\left\|R_{T, 2}\left(u_{\mathscr{T}}^{*}, p_{\mathscr{T}}^{*}\right)\right\|_{L^{2}(T)}^{2}+\sum_{F \subset \partial T} h_{F}^{3}\left\|J_{F, 2}\left(\sigma_{\mathscr{T}}^{*}\right)\right\|_{L^{2}(F)}^{2} .
\end{aligned}
$$

We defer the derivation of the a posteriori error estimator $\eta_{\mathscr{T}}\left(\sigma_{\mathscr{T}}^{*}, u_{\mathscr{T}}^{*}, U_{\mathscr{T}}^{*}, p_{\mathscr{T}}^{*}, P_{\mathscr{T}}^{*}, \mathscr{M}_{\mathscr{T}}\right)$ to Section 3.3 below. The notation $\mathscr{M}_{\mathscr{T}}$ will be omitted whenever $\mathscr{M}_{\mathscr{T}}=\mathscr{T}$. Note that the estimator $\eta_{\mathscr{T}}$ depends only on the discrete solutions $\left(\sigma_{\mathscr{T}}^{*}, u_{\mathscr{T}}^{*}, U_{\mathscr{T}}^{*}, p_{\mathscr{T}}^{*}, P_{\mathscr{T}}^{*}\right)$ and the given problem data (e.g., impedance coefficients $\left\{z_{l}\right\}_{l=1}^{L}$ ), and all the quantities involved in $\eta_{\mathscr{T}}$ are computable. Further, the regularization parameter $\alpha$ enters the estimator only through the face residual $J_{F, 2}\left(\sigma_{\mathscr{T}}^{*}\right)$. It will be shown in Section 4.2 that this error estimator is sufficient for the convergence of the resulting adaptive algorithm.

Now we can formulate an adaptive algorithm for the EIT inverse problem, cf. Algorithm 1. Below we indicate the dependence on the triangulation $\mathscr{T}_{k}$ by the iteration number $k$ in the subscript.

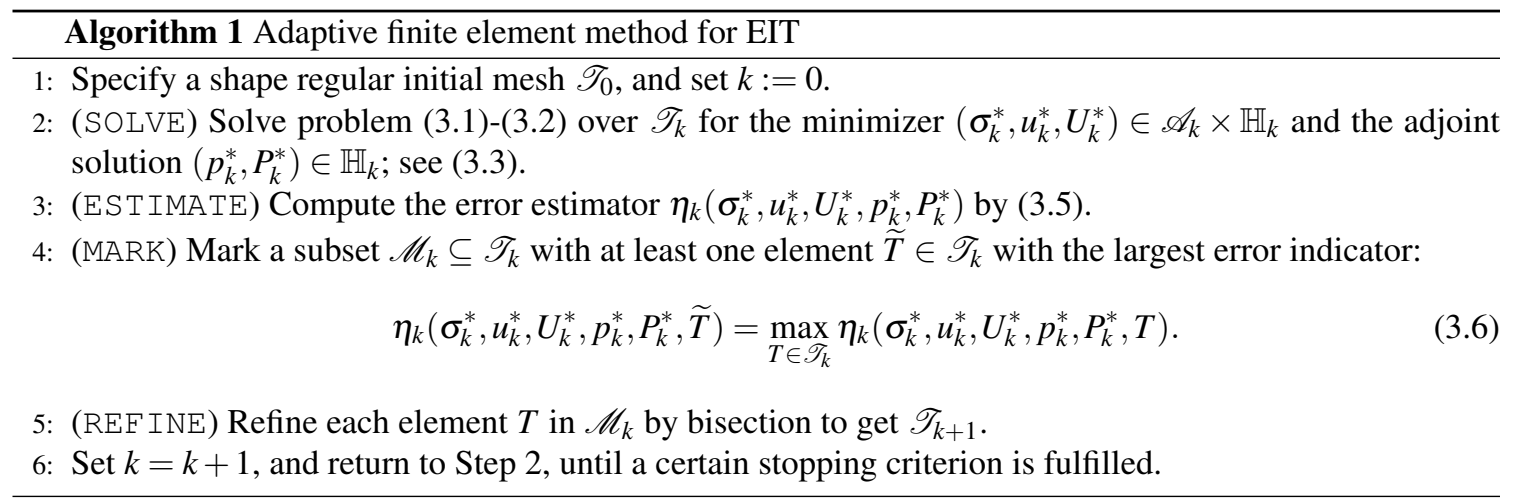

REMARK 3.2 The solver in the module SOLVE can be either a (projected) gradient descent method or iteratively regularized Gauss-Newton method, each equipped with a suitable step size selection rule. 
REMARK 3.3 Assumption (3.6) in the module MARK is fairly general, and it covers several commonly used collective marking strategies, e.g., maximum strategy, equidistribution, modified equidistribution strategy, and Dörfler's strategy (Siebert, 2011, pp. 962). Our convergence analysis in Section 4 covers all these marking strategies. In the module MARK, one may also consider separate marking. The motivation is to be able to neglect data oscillations, which have no importance for sufficiently fine meshes. Numerically, this adds little computational overheads, since the module SOLVE is the most expensive step at each iteration.

Last, we give an important geometric observation on the mesh sequence $\left\{\mathscr{T}_{k}\right\}$ and a stability result on error indicators $\eta_{k, 1}\left(\sigma_{k}^{*}, u_{k}^{*}, U_{k}^{*}\right), \eta_{k, 2}\left(\sigma_{k}^{*}, p_{k}^{*}, P_{k}^{*}\right)$ and $\eta_{k, 3}\left(\sigma_{k}^{*}, u_{k}^{*}, p_{k}^{*}\right)$ given in Algorithm 1. Let

$$
\mathscr{T}_{k}^{+}:=\bigcap_{l \geqslant k} \mathscr{T}_{l}, \quad \mathscr{T}_{k}^{0}:=\mathscr{T}_{k} \backslash \mathscr{T}_{k}^{+}, \quad \Omega_{k}^{+}:=\bigcup_{T \in \mathscr{T}_{k}^{+}} D_{T}, \quad \Omega_{k}^{0}:=\bigcup_{T \in \mathscr{T}_{k}^{0}} D_{T} .
$$

That is, the set $\mathscr{T}_{k}^{+}$consists of all elements not refined after the $k$-th iteration while all elements in $\mathscr{T}_{k}^{0}$ are refined at least once after the $k$-th iteration. Clearly, $\mathscr{T}_{l}^{+} \subset \mathscr{T}_{k}^{+}$for $l<k$. We also define a mesh-size function $h_{k}: \bar{\Omega} \rightarrow \mathbb{R}^{+}$almost everywhere by $h_{k}(x)=h_{T}$ for $x$ in the interior of an element $T \in \mathscr{T}_{k}$ and $h_{k}(x)=h_{F}$ for $x$ in the relative interior of an edge $F \in \mathscr{F}_{k}$. It has the following important property in the region of $\Omega$ involving marked elements (Siebert, 2011, Corollary 3.3).

LEMMA 3.4 Let $\chi_{k}^{0}$ be the characteristic function of $\Omega_{k}^{0}$. Then $\lim _{k \rightarrow \infty}\left\|h_{k} \chi_{k}^{0}\right\|_{L^{\infty}(\Omega)}=0$.

The next result gives preliminary bounds on the a posteriori error estimators. Note that only the constant $c$ for the estimator $\eta_{k, 3}$ depends on the regularization parameter $\alpha$, via the face residuals $J_{F, 2}\left(\sigma_{k}^{*}\right)$, and all the constants can be naturally made independent of $\alpha$, if desired.

LEMMA 3.5 Let the sequence of discrete solutions $\left\{\left(\sigma_{k}^{*}, u_{k}^{*}, U_{k}^{*}, p_{k}^{*}, P_{k}^{*}\right)\right\}$ be generated by Algorithm 1 . Then for each $T \in \mathscr{T}_{k}$ with its face $F$, there hold

$$
\begin{aligned}
& \eta_{k, 1}^{2}\left(\sigma_{k}^{*}, u_{k}^{*}, U_{k}^{*}, T\right) \leqslant c\left(\left\|\nabla u_{k}^{*}\right\|_{L^{2}\left(D_{T}\right)}^{2}+h_{F}\left\|u_{k}^{*}-U_{k, l}^{*}\right\|_{L^{2}\left(F \cap e_{l}\right)}^{2}\right), \\
& \eta_{k, 2}^{2}\left(\sigma_{k}^{*}, p_{k}^{*}, P_{k}^{*}, T\right) \leqslant c\left(\left\|\nabla p_{k}^{*}\right\|_{L^{2}\left(D_{T}\right)}^{2}+h_{F}\left\|p_{k}^{*}-P_{k, l}^{*}\right\|_{L^{2}\left(F \cap e_{l}\right)}^{2}\right), \\
& \eta_{k, 3}^{2}\left(\sigma_{k}^{*}, u_{k}^{*}, p_{k}^{*}, T\right) \leqslant c\left(h_{T}^{4-d}\left\|\nabla u_{k}^{*}\right\|_{L^{2}(T)}^{2}\left\|\nabla p_{k}^{*}\right\|_{L^{2}(T)}^{2}+h_{T}^{2}\left\|\nabla \sigma_{k}^{*}\right\|_{L^{2}\left(D_{T}\right)}^{2}\right),
\end{aligned}
$$

where $e_{l}$ denotes the electrode intersecting with the element $T \in \mathscr{T}_{k}$.

Proof. We only prove the third estimate, and the first two follow analogously. By the inverse estimates and the trace theorem, the local quasi-uniformity of $\mathscr{T}_{k}$ yields

$$
\begin{aligned}
& h_{T}^{4}\left\|\nabla u_{k}^{*} \cdot \nabla p_{k}^{*}\right\|_{L^{2}(T)}^{2} \leqslant c h_{T}^{4-d}\left\|\nabla u_{k}^{*} \cdot \nabla p_{k}^{*}\right\|_{L^{1}(T)}^{2} \leqslant c h_{T}^{4-d}\left\|\nabla u_{k}^{*}\right\|_{L^{2}(T)}^{2}\left\|\nabla p_{k}^{*}\right\|_{L^{2}(T)}^{2}, \\
& \sum_{F \subset \partial T} h_{F}^{3}\left\|J_{F, 2}\left(\sigma_{k}^{*}\right)\right\|_{L^{2}(F)}^{2} \leqslant c h_{T}^{2}\left\|\nabla \sigma_{k}^{*}\right\|_{L^{2}\left(D_{T}\right)}^{2} .
\end{aligned}
$$

\subsection{Derivation of a posteriori error estimators}

Now we motivate the a posteriori error estimator $\eta_{\mathscr{T}}$ defined in (3.5) underlying the module EST IMATE of Algorithm 1. The algorithm generates a sequence of discrete solutions $\left\{\left(\sigma_{k}^{*}, u_{k}^{*}, U_{k}^{*}, p_{k}^{*}, P_{k}^{*}\right)\right\}$ in a sequence of finite element spaces $\left\{V_{k}\right\}$ and discrete admissible sets $\left\{\mathscr{A}_{k}\right\}$ over a sequence of meshes $\left\{\mathscr{T}_{k}\right\}$. Naturally, some arguments in the a posteriori error estimation for direct problems will be employed. We shall need 
the following results on the Lagrange interpolation operator $I_{k}: H^{2}(\Omega) \rightarrow V_{k}$ (Ciarlet (2002)) and the ScottZhang interpolation operator $I_{k}^{s z}: H^{1}(\Omega) \rightarrow V_{k}$ (Scott \& Zhang (1990)) over the triangulation $\mathscr{T}_{k}$.

LEMmA 3.6 Let $\omega_{F}$ is the union of elements with $F$ as a face. For any $T \in \mathscr{T}_{k}$ and any $F \in \mathscr{F}_{k}$,

$$
\begin{aligned}
& \left\|v-I_{k} v\right\|_{L^{2}(T)} \leqslant c h_{T}^{2}\|v\|_{H^{2}(T)}, \quad\left\|v-I_{k} v\right\|_{L^{2}(F)} \leqslant c h_{T}^{3 / 2}\|v\|_{H^{2}\left(\omega_{F}\right)}, \\
& \left\|v-I_{k}^{s z} v\right\|_{L^{2}(T)} \leqslant c h_{T}\|v\|_{H^{1}\left(D_{T}\right)}, \quad\left\|v-I_{k}^{s z} v\right\|_{L^{2}(F)} \leqslant c h_{T}^{1 / 2}\|v\|_{H^{1}\left(D_{F}\right)} .
\end{aligned}
$$

To motivate the error estimator $\eta_{\mathscr{T}}$, we begin with two auxiliary problems: find $\left(\widetilde{u}\left(\sigma_{k}^{*}\right), \widetilde{U}\left(\sigma_{k}^{*}\right)\right) \in \mathbb{H}$ and $\left(\widetilde{p}\left(\sigma_{k}^{*}\right), \widetilde{P}\left(\sigma_{k}^{*}\right)\right) \in \mathbb{H}$ such that

$$
\begin{aligned}
& a\left(\sigma_{k}^{*},(\widetilde{u}, \widetilde{U}),(v, V)\right)=\langle I, V\rangle \quad \forall(v, V) \in \mathbb{H}, \\
& a\left(\sigma_{k}^{*},(\widetilde{p}, \widetilde{P}),(v, V)\right)=\left\langle\widetilde{U}-U^{\delta}, V\right\rangle \quad \forall(v, V) \in \mathbb{H} .
\end{aligned}
$$

The first line in (3.3) is actually the finite element scheme of (3.7) over the triangulation $\mathscr{T}_{k}$. Hence, the standard a posteriori error analysis for forward problems can be applied. By setting $v_{k}=I_{k}^{s z} v \in V_{k}$ in the first line in (3.3) for any $(v, V) \in \mathbb{H}$, applying elementwise integration by parts and Lemma 3.6, there hold

$$
\begin{aligned}
a\left(\sigma_{k}^{*},\left(\widetilde{u}-u_{k}^{*}, \widetilde{U}-U_{k}^{*}\right),(v, V)\right) & =\langle I, V\rangle-\left(\sigma_{k}^{*} \nabla u_{k}^{*}, \nabla v\right)-\sum_{l=1}^{L} z_{l}^{-1}\left(u_{k}^{*}-U_{k, l}^{*}, v-V_{l}\right)_{L^{2}\left(e_{l}\right)} \\
& =\left(\sigma_{k}^{*} \nabla u_{k}^{*}, \nabla\left(I_{k}^{s z} v-v\right)\right)+\sum_{l=1}^{L} z_{l}^{-1}\left(u_{k}^{*}-U_{k, l}^{*}, I_{k}^{s z} v-v\right)_{L^{2}\left(e_{l}\right)} \\
& \leqslant c\left(\sum_{T \in \mathscr{T} k} \eta_{k, 1}^{2}\left(\sigma_{k}^{*}, u_{k}^{*}, U_{k}^{*}, T\right)\right)^{1 / 2}\|v\|_{H^{1}(\Omega)} .
\end{aligned}
$$

Taking $(v, V)=\left(\widetilde{u}-u_{k}^{*}, \widetilde{U}-U_{k}^{*}\right) \in \mathbb{H}$ and using Lemma 2.1 yield

$$
\left\|\left(\widetilde{u}-u_{k}^{*}, \widetilde{U}-U_{k}^{*}\right)\right\|_{\mathbb{H}, *} \leqslant c\left(\sum_{T \in \mathscr{T}_{k}} \eta_{k, 1}^{2}\left(\sigma_{k}^{*}, u_{k}^{*}, U_{k}^{*}, T\right)\right)^{1 / 2} .
$$

Further, from the first equation in (2.5) and (3.7) we find for any $(v, V) \in \mathbb{H}$

$$
a\left(\sigma_{k}^{*},\left(u^{*}-\widetilde{u}, U^{*}-\widetilde{U}\right),(v, V)\right)=\left(\left(\sigma_{k}^{*}-\sigma^{*}\right) \nabla u^{*}, \nabla v\right) \leqslant\left\|\left(\sigma^{*}-\sigma_{k}^{*}\right) \nabla u^{*}\right\|_{L^{2}(\Omega)}\|\nabla v\|_{L^{2}(\Omega)} .
$$

Consequently,

$$
\left\|\left(u^{*}-\widetilde{u}, U^{*}-\widetilde{U}\right)\right\|_{\mathbb{H}, *} \leqslant c\left\|\left(\sigma^{*}-\sigma_{k}^{*}\right) \nabla u^{*}\right\|_{L^{2}(\Omega)} .
$$

Likewise, for $\left(p^{*}-p_{k}^{*}, P^{*}-P_{k}^{*}\right)$, we appeal to the second equation in the discrete optimality system (3.3) and the auxiliary problem (3.8) to deduce

$$
\begin{aligned}
a\left(\sigma_{k}^{*},\left(\widetilde{p}-p_{k}^{*}, \widetilde{P}-P_{k}^{*}\right),(v, V)\right)=\left\langle\widetilde{U}-U^{\delta}, V\right\rangle-a\left(\sigma_{k}^{*},\left(p_{k}^{*}, P_{k}^{*}\right),(v, V)\right) \\
=\left\langle\widetilde{U}-U_{k}^{*}, V\right\rangle+\left\langle U_{k}^{*}-U^{\delta}, V\right\rangle-a\left(\sigma_{k}^{*},\left(p_{k}^{*}, P_{k}^{*}\right),(v, V)\right) \\
=\left\langle\widetilde{U}-U_{k}^{*}, V\right\rangle+\left(\sigma_{k}^{*} \nabla p_{k}^{*}, \nabla\left(I_{k}^{s z} v-v\right)\right)+\sum_{l=1}^{L} z_{l}^{-1}\left(p_{k}^{*}-P_{k, l}^{*}, I_{k}^{s z} v-v\right)_{L^{2}\left(e_{l}\right)} \\
\quad \leqslant c\left(\left(\sum_{T \in \mathscr{T}_{k}} \eta_{k, 2}^{2}\left(\sigma_{k}^{*}, p_{k}^{*}, P_{k}^{*}, T\right)\right)^{1 / 2}+\left\|\widetilde{U}-U_{k}^{*}\right\|\right)\|(v, V)\|_{\mathbb{H}, *},
\end{aligned}
$$


and further

$$
\begin{aligned}
a\left(\sigma_{k}^{*},\left(p^{*}-\widetilde{p}, P^{*}-\widetilde{P}\right),(v, V)\right) & =\left(\left(\sigma_{k}^{*}-\sigma^{*}\right) \nabla p^{*}, \nabla v\right)+\left\langle U^{*}-\widetilde{U}, V\right\rangle \\
& \leqslant\left(\left\|\left(\sigma^{*}-\sigma_{k}^{*}\right) \nabla p^{*}\right\|_{L^{2}(\Omega)}+\left\|U^{*}-\widetilde{U}\right\|\right)\|(v, V)\|_{\mathbb{H}, *}
\end{aligned}
$$

which, together with (3.9) and (3.10) and Lemma 2.1, implies

$$
\begin{aligned}
\left\|\left(p^{*}-p_{k}^{*}, P^{*}-P_{k}^{*}\right)\right\|_{\mathbb{H}, *} \leqslant & c\left(\left(\sum_{T \in \mathscr{T}_{k}} \eta_{k, 1}^{2}\left(\sigma_{k}^{*}, u_{k}^{*}, U_{k}^{*}, T\right)+\eta_{k, 2}^{2}\left(\sigma_{k}^{*}, p_{k}^{*}, P_{k}^{*}, T\right)\right)^{1 / 2}\right. \\
& \left.+\left\|\left(\sigma^{*}-\sigma_{k}^{*}\right) \nabla u^{*}\right\|_{L^{2}(\Omega)}+\left\|\left(\sigma^{*}-\sigma_{k}^{*}\right) \nabla p^{*}\right\|_{L^{2}(\Omega)}\right) .
\end{aligned}
$$

In view of (3.9)-(3.11), the estimators $\eta_{k, 1}$ and $\eta_{k, 2}$ can bound $\left(u^{*}-u_{k}^{*}, U^{*}-U_{k}^{*}\right)$ and $\left(p^{*}-p_{k}^{*}, P^{*}-P_{k}^{*}\right)$ from above up to the terms $\left\|\left(\sigma^{*}-\sigma_{k}^{*}\right) \nabla u^{*}\right\|_{L^{2}(\Omega)}$ and $\left\|\left(\sigma^{*}-\sigma_{k}^{*}\right) \nabla p^{*}\right\|_{L^{2}(\Omega)}$, which are not computable but asymptotically vanishing, provided that $\sigma_{k}^{*} \rightarrow \sigma^{*}$ pointwise. This motivates our choice of a computable upper bound for $\sigma^{*}-\sigma_{k}^{*}$, upon discarding the uncomputable terms.

To bound the term $\left\|\nabla\left(\sigma_{k}^{*}-\sigma^{*}\right)\right\|_{L^{2}(\Omega)}$, we appeal to the variational inequalities in (2.5) and (3.3). Since $I_{k} \mu \in \mathscr{A}_{k}$ for any $\mu \in \mathscr{A} \cap C^{\infty}(\bar{\Omega})$, we deduce

$$
\begin{aligned}
\alpha\left\|\nabla\left(\sigma^{*}-\sigma_{k}^{*}\right)\right\|_{L^{2}(\Omega) \leqslant}^{2} & \alpha\left(\nabla \sigma_{k}^{*}, \nabla\left(\sigma_{k}^{*}-\sigma^{*}\right)\right)-\left(\left(\sigma_{k}^{*}-\sigma^{*}\right) \nabla u^{*}, \nabla p^{*}\right) \\
= & \alpha\left(\nabla \sigma_{k}^{*}, \nabla\left(\sigma_{k}^{*}-\sigma^{*}\right)\right)-\left(\left(\sigma_{k}^{*}-\sigma^{*}\right) \nabla u_{k}^{*}, \nabla p_{k}^{*}\right) \\
& +\left(\nabla u_{k}^{*} \cdot \nabla p_{k}^{*}-\nabla u^{*} \cdot \nabla p^{*}, \sigma_{k}^{*}-\sigma^{*}\right) \\
\leqslant & \alpha\left(\nabla \sigma_{k}^{*}, \nabla\left(I_{k} \mu-\sigma^{*}\right)\right)-\left(\left(I_{k} \mu-\sigma^{*}\right) \nabla u_{k}^{*}, \nabla p_{k}^{*}\right) \\
& +\left(\nabla u_{k}^{*} \cdot \nabla p_{k}^{*}-\nabla u^{*} \cdot \nabla p^{*}, \sigma_{k}^{*}-\sigma^{*}\right) \\
= & \alpha\left(\nabla \sigma_{k}^{*}, \nabla\left(I_{k} \mu-\mu\right)\right)-\left(\nabla u_{k}^{*}, \nabla p_{k}^{*}\left(I_{k} \mu-\mu\right)\right) \\
& +\left(\nabla u_{k}^{*} \cdot \nabla p_{k}^{*}-\nabla u^{*} \cdot \nabla p^{*}, \sigma_{k}^{*}-\sigma^{*}\right) \\
& +\alpha\left(\nabla \sigma_{k}^{*}, \nabla\left(\mu-\sigma^{*}\right)\right)-\left(\left(\mu-\sigma^{*}\right) \nabla u_{k}^{*}, \nabla p_{k}^{*}\right):=\mathrm{I}+\mathrm{II}+\mathrm{III} .
\end{aligned}
$$

Now Lemma 3.6 and elementwise integration by parts yield

$$
|\mathrm{I}| \leqslant c\left(\sum_{T \in \mathscr{T}_{k}} \eta_{k, 3}^{2}\left(\sigma_{k}^{*}, u_{k}^{*}, p_{k}^{*}, T\right)\right)^{1 / 2}\|\mu\|_{H^{2}(\Omega)} \quad \forall \mu \in \mathscr{A} \cap C^{\infty}(\bar{\Omega}) .
$$

By the minimizing property of $\sigma_{k}^{*}$ for $J_{k}(\cdot),\left\|\nabla \sigma_{k}^{*}\right\|_{L^{2}(\Omega)}$ is bounded. Then the estimate (3.4) and the density of $\mathscr{A} \cap C^{\infty}(\bar{\Omega})$ in $\mathscr{A}$ ensure that the term III can be made arbitrarily small. For the term II, we have

$$
\begin{aligned}
|\mathrm{II}| & =\left|\left(\nabla u_{k}^{*} \cdot \nabla p_{k}^{*}-\nabla u_{k}^{*} \cdot \nabla p^{*}+\nabla u_{k}^{*} \cdot \nabla p^{*}-\nabla u^{*} \cdot \nabla p^{*}, \sigma^{k}-\sigma^{*}\right)\right| \\
& \leqslant\left\|\nabla\left(p_{k}^{*}-p^{*}\right)\right\|_{L^{2}(\Omega)}\left\|\left(\sigma_{k}^{*}-\sigma^{*}\right) \nabla u_{k}^{*}\right\|_{L^{2}(\Omega)}+\left\|\nabla\left(u_{k}^{*}-u^{*}\right)\right\|_{L^{2}(\Omega)}\left\|\left(\sigma_{k}^{*}-\sigma^{*}\right) \nabla p^{*}\right\|_{L^{2}(\Omega)},
\end{aligned}
$$

which are expected to be higher order terms. Upon discarding the uncomputable terms $\left\|\left(\sigma^{*}-\sigma_{k}^{*}\right) \nabla u^{*}\right\|_{L^{2}(\Omega)}$ and $\left\|\left(\sigma^{*}-\sigma_{k}^{*}\right) \nabla p^{*}\right\|_{L^{2}(\Omega)}$ in (3.10)-(3.11) and the nonlinear term II, we get all computable quantities in (3.9), (3.11) and (3.12), which are exactly the a posteriori error estimator $\eta_{k}$ defined in (3.5). Thus we may view it as a reliable upper bound for the error and employ it in the module ESTIMATE to drive the adaptive refinement process. Moreover the derivation of (3.12) suggests itself a natural way to handle the variational inequality in (2.5) in the convergence analysis, which will be presented in Section 4 below. 


\section{Convergence analysis}

In this section, we shall establish the main theoretical result of this work, the convergence of Algorithm 1 , namely the sequence of discrete solutions $\left\{\left(\sigma_{k}^{*}, u_{k}^{*}, U_{k}^{*}, p_{k}^{*}, P_{k}^{*}\right)\right\}$ to the optimality system (3.3) generated by Algorithm 1, contains a subsequence converging in $H^{1}(\Omega) \times \mathbb{H} \times \mathbb{H}$ to a solution to the optimality system (2.5). The main technical difficulty lies in the lack of density of the adaptively generated FE space $V_{k}$ in the space $H^{1}(\Omega)$. To overcome the challenge, the proof is carried out in two steps. In the first step (Section 4.1), we analyze a "limiting" optimization problem posed over a limiting set induced by $\left\{\mathscr{A}_{k}\right\}$, and show that the sequence of discrete solutions contains a convergent subsequence to a minimizer to the limiting problem. In the second step (Section 4.2), we show that the solution to the optimality system for the limiting problem actually solves the optimality system (2.5). It is worth noting that all the proofs in Section 4.1 only depends on the nestedness of finite element spaces $\left\{V_{k}\right\}$ and discrete admissible sets $\left\{\mathscr{A}_{k}\right\}$, and the error estimator (3.5) and the marking assumption (3.6) are used only in Section 4.2.

\subsection{Limiting optimization problem}

For the sequences $\left\{\mathbb{H}_{k}\right\}$ and $\left\{\mathscr{A}_{k}\right\}$ generated by Algorithm 1, we define a limiting finite element space $\mathbb{H}_{\infty}$ and a limiting admissible set $\mathscr{A}_{\infty}$ respectively by

$$
\mathbb{H}_{\infty}:=\overline{\bigcup_{k \geqslant 0} \mathbb{H}_{k}} \text { (in } \mathbb{H}, * \text {-norm) and } \mathscr{A}_{\infty}:=\overline{\bigcup_{k \geqslant 0} \mathscr{A}_{k}}\left(\text { in } H^{1}(\Omega) \text {-norm }\right) .
$$

It is easy to see that $\mathbb{H}_{\infty}$ is a closed subspace of $\mathbb{H}$. For the set $\mathscr{A}_{\infty}$, we have the following lemma.

LEMMA $4.1 \mathscr{A}_{\infty}$ is a closed convex subset of $\mathscr{A}$.

Proof. The definition of $\mathscr{A}_{\infty}$ implies its strong closedness. For any $\mu$ and $v$ in $\mathscr{A}_{\infty}$, there exist two sequences $\left\{\mu_{k}\right\}$ and $\left\{v_{k}\right\} \subset \bigcup_{k \geqslant 0} \mathscr{A}_{k}$ such that $\mu_{k} \rightarrow \mu$ and $v_{k} \rightarrow v$ in $H^{1}(\Omega)$. The convexity of the set $\mathscr{A}_{k}$ implies $\left\{t \mu_{k}+(1-t) v_{k}\right\} \subset \bigcup_{k \geqslant 0} \mathscr{A}_{k}$ for any $t \in(0,1)$. Then $t \mu_{k}+(1-t) v_{k} \rightarrow t \mu+(1-t) v$ in $H^{1}(\Omega)$, i.e. $t \mu+(1-t) v \in \mathscr{A}_{\infty}$ for any $t \in(0,1)$. Hence $\mathscr{A}_{\infty}$ is convex. Moreover, we have $\mu_{k} \rightarrow \mu$ a.e. in $\Omega$ after (possibly) passing to a subsequence, which, along with the constraint $\lambda \leqslant \mu_{k} \leqslant \lambda^{-1}$ a.e. in $\Omega$, indicates that $\lambda \leqslant \mu \leqslant \lambda^{-1}$ a.e. in $\Omega$. Lastly, the fact that $\mathscr{A}_{\infty} \subset H^{1}(\Omega)$ concludes $\mathscr{A}_{\infty} \subset \mathscr{A}$.

Over the limiting set $\mathscr{A}_{\infty}$, we introduce a limiting minimization problem:

$$
\min _{\sigma_{\infty} \in \mathscr{A}_{\infty}}\left\{J_{\infty}\left(\sigma_{\infty}\right)=\frac{1}{2}\left\|U_{\infty}\left(\sigma_{\infty}\right)-U^{\delta}\right\|^{2}+\frac{\alpha}{2}\left\|\nabla \sigma_{\infty}\right\|_{L^{2}(\Omega)}^{2}\right\}
$$

where $\left(u_{\infty}, U_{\infty}\right) \equiv\left(u_{\infty}\left(\sigma_{\infty}\right), U_{\infty}\left(\sigma_{\infty}\right)\right) \in \mathbb{H}_{\infty}$ satisfies the variational problem:

$$
a\left(\sigma_{\infty},\left(u_{\infty}, U_{\infty}\right),(v, V)\right)=\langle I, V\rangle \quad \forall(v, V) \in \mathbb{H}_{\infty} .
$$

By Lemma 2.1 and Lax-Milgram theorem, the limiting variational problem (4.2) is well-posed for any fixed $\sigma_{\infty} \in \mathscr{A}_{\infty}$. The next result shows the existence of a minimizer to the limiting problem (4.1)-(4.2).

THEOREM 4.2 There exists at least one minimizer to problem (4.1)-(4.2).

Proof. It is clear that $\inf J_{\infty}(\sigma)$ is finite over $\mathscr{A}_{\infty}$, so there exists a minimizing sequence $\left\{\sigma^{n}\right\} \subset \mathscr{A}_{\infty}$, i.e.,

$$
\lim _{n \rightarrow \infty} J_{\infty}\left(\sigma^{n}\right)=\inf _{\sigma \in \mathscr{A}_{\infty}} J_{\infty}(\sigma)
$$

Thus, the sequence $\left\{\sigma^{n}\right\}$ is uniformly bounded in $H^{1}(\Omega)$, and by Sobolev embedding theorem and Lemma 4.1, there exists a subsequence, relabeled as $\left\{\sigma^{n}\right\}$, and some $\sigma^{*} \in \mathscr{A}_{\infty}$ such that $\sigma^{n} \rightarrow \sigma^{*}$ weakly in $H^{1}(\Omega)$, 
$\sigma^{n} \rightarrow \sigma^{*}$ a.e. in $\Omega$. By taking $\sigma_{\infty}=\sigma^{n} \in \mathscr{A}_{\infty}$ in (4.2), then $\left(u^{n}, U^{n}\right) \equiv\left(u^{n}\left(\sigma^{n}\right), U^{n}\left(\sigma^{n}\right)\right) \in \mathbb{H}_{\infty} \subset \mathbb{H}_{\text {satisfies }}$

$$
a\left(\sigma^{n},\left(u^{n}, U^{n}\right),(v, V)\right)=\langle I, V\rangle \quad \forall(v, V) \in \mathbb{H}_{\infty}
$$

Then by Lemma 2.1, $\left\{\left(u^{n}, U^{n}\right)\right\}$ is uniformly bounded in $\mathbb{H}$, which gives a subsequence, also denoted by $\left\{\left(u^{n}, U^{n}\right)\right\}$, and some $\left(u^{*}, U^{*}\right) \in \mathbb{H}_{\infty}$ such that

$$
\left(u^{n}, U^{n}\right) \rightarrow\left(u^{*}, U^{*}\right) \quad \text { weakly in } \mathbb{H} \quad \text { and } \quad u^{n} \rightarrow u^{*} \quad \text { in } L^{2}(\Gamma) .
$$

We claim that $\left(u^{*}, U^{*}\right)=\left(u^{*}\left(\sigma^{*}\right), U^{*}\left(\sigma^{*}\right)\right) \in \mathbb{H}_{\infty}$. To this end, first we observe the splitting

$$
\left(\sigma^{n} \nabla u^{n}, \nabla v\right)=\left(\left(\sigma^{n}-\sigma^{*}\right) \nabla u^{n}, \nabla v\right)+\left(\sigma^{*} \nabla u^{n}, \nabla v\right)
$$

The pointwise convergence of the sequence $\left\{\sigma^{n}\right\}$, Lebesgue's dominated convergence theorem (Evans \& Gariepy (1992)) and the uniform boundedness of $\left\{u^{n}\right\}$ in $H^{1}(\Omega)$ imply that

$$
\left|\left(\left(\sigma^{n}-\sigma^{*}\right) \nabla u^{n}, \nabla v\right)\right| \leqslant\left\|\nabla u^{n}\right\|_{L^{2}(\Omega)}\left\|\left(\sigma^{n}-\sigma^{*}\right) \nabla v\right\|_{L^{2}(\Omega)} \rightarrow 0 .
$$

This and the weak convergence of $\left\{u^{n}\right\}$ in $H^{1}(\Omega)$ give

$$
\left(\sigma^{n} \nabla u^{n}, \nabla v\right)_{L^{2}(\Omega)} \rightarrow\left(\sigma^{*} \nabla u^{*}, \nabla v\right)_{L^{2}(\Omega)}
$$

Then by (4.4), we obtain

$$
\left(u^{n}-U_{l}^{n}, v-V_{l}\right)_{L^{2}\left(e_{l}\right)} \rightarrow\left(u^{*}-U_{l}^{*}, v-V_{l}\right)_{L^{2}\left(e_{l}\right)} .
$$

Upon taking into account these relations, we deduce

$$
a\left(\sigma^{*},\left(u^{*}, U^{*}\right),(v, V)\right)=\langle I, V\rangle \quad \forall(v, V) \in \mathbb{H}_{\infty},
$$

i.e., the desired claim $\left(u^{*}, U^{*}\right)=\left(u^{*}\left(\sigma^{*}\right), U^{*}\left(\sigma^{*}\right)\right) \in \mathbb{H}_{\infty}$. This and the weak lower semicontinuity of the norm imply that $\sigma^{*}$ is a minimizer of $J_{\infty}(\cdot)$ over $\mathscr{A}_{\infty}$, completing the proof of the theorem.

The preceding proof together with the uniqueness of the solution to (4.2) and the standard subsequence argument yields the following weak continuity result.

LEMmA 4.3 Let the sequence $\left\{\sigma_{k}\right\} \subset \bigcup_{k \geqslant 0} \mathscr{A}_{k}$ converge to some $\sigma^{*} \in \mathscr{A}_{\infty}$ weakly in $H^{1}(\Omega)$ and let the solution to (4.2) with $\sigma_{\infty}=\sigma^{*}$ be $\left(u\left(\sigma^{*}\right), U\left(\sigma^{*}\right)\right) \in \mathbb{H}_{\infty}$. Then the sequence of solutions $\left\{\left(u_{k}\left(\sigma_{k}\right), U_{k}\left(\sigma_{k}\right)\right)\right\} \subset$ $\bigcup_{k \geqslant 0} \mathbb{H}_{k}$ to (3.1) over $\mathscr{T}_{k}$ satisfies

$$
\left(u_{k}\left(\sigma_{k}\right), U_{k}\left(\sigma_{k}\right)\right) \rightarrow\left(u\left(\sigma^{*}\right), U\left(\sigma^{*}\right)\right) \quad \text { weakly in } \mathbb{H}
$$

Now we analyze the limiting behavior of the sequence $\left\{\left(\sigma_{k}^{*}, u_{k}^{*}, U_{k}^{*}\right)\right\}$ generated by Algorithm 1: It contains a subsequence converging in $H^{1}(\Omega) \times \mathbb{H}$ to a minimizer of the limiting problem (4.1)-(4.2). This result will play a crucial role in the convergence analysis in Section 4.2.

THEOREM 4.4 Let $\left\{\mathscr{A}_{k} \times \mathbb{H}_{k}\right\}$ be a sequence of discrete admissible sets and finite element spaces generated by Algorithm 1. Then the sequence of discrete solutions $\left\{\left(\sigma_{k}^{*}, u_{k}^{*}, U_{k}^{*}\right)\right\}$ to problem (3.2) has a subsequence $\left\{\left(\sigma_{k_{m}}^{*}, u_{k_{m}}^{*}, U_{k_{m}}^{*}\right)\right\}$ converging to a minimizer $\left(\sigma_{\infty}^{*}, u_{\infty}^{*}, U_{\infty}^{*}\right)$ to problem (4.1)-(4.2) in the sense that

$$
\sigma_{k_{m}}^{*} \rightarrow \sigma_{\infty}^{*} \quad \text { in } H^{1}(\Omega), \quad \sigma_{k_{m}}^{*} \rightarrow \sigma_{\infty}^{*} \quad \text { a.e. in } \Omega, \quad\left(u_{k_{m}}^{*}, U_{k_{m}}^{*}\right) \rightarrow\left(u_{\infty}^{*}, U_{\infty}^{*}\right) \quad \text { in } \mathbb{H} .
$$


Proof. Since the function $\sigma \equiv 1 \in \mathscr{A}_{k}$ for all $k$ and $J_{k}\left(\sigma_{k}^{*}\right)$ attains its minimum at $\sigma_{k}^{*} \in \mathscr{A}_{k}$, the sequence $\left\{\sigma_{k}^{*}\right\}$ is uniformly bounded in $H^{1}(\Omega)$. By Sobolev embedding theorem, there exists a subsequence $\left\{\sigma_{k_{m}}^{*}\right\}$ and some $\sigma_{\infty}^{*} \in \mathscr{A}_{\infty}$ such that $\sigma_{k_{m}}^{*} \rightarrow \sigma_{\infty}^{*}$ weakly in $H^{1}(\Omega), \sigma_{k_{m}}^{*} \rightarrow \sigma_{\infty}^{*}$ a.e. in $\Omega$. By Lemma 4.3, there exists a subsequence of $\left\{\left(u_{k_{m}}^{*}, U_{k_{m}}^{*}\right)\right\}$ such that

$$
\left(u_{k_{m}}^{*}, U_{k_{m}}^{*}\right) \rightarrow\left(u_{\infty}^{*}\left(\sigma_{\infty}^{*}\right), U_{\infty}^{*}\left(\sigma_{\infty}^{*}\right)\right) \quad \text { weakly in } \mathbb{H},
$$

where $\left(u_{\infty}^{*}\left(\sigma_{\infty}^{*}\right), U_{\infty}^{*}\left(\sigma_{\infty}^{*}\right)\right)$ solves (4.2) with $\sigma_{\infty}=\sigma_{\infty}^{*}$. We claim that $\sigma_{\infty}^{*}$ is a minimizer to $J_{\infty}$ over $\mathscr{A}_{\infty}$. For any $\sigma \in \mathscr{A}_{\infty}$, the definition of $\mathscr{A}_{\infty}$ ensures the existence of a sequence $\left\{\sigma_{k}\right\} \subset \bigcup_{k \geqslant 0} \mathscr{A}_{k}$ such that $\sigma_{k} \rightarrow \sigma$ in $H^{1}(\Omega)$. By Lemma 4.3, the sequence of solutions $\left(u_{k}\left(\sigma_{k}\right), U_{k}\left(\sigma_{k}\right)\right)$ to problem (3.1) over $\mathscr{T}_{k}$ satisfies

$$
\left(u_{k}\left(\sigma_{k}\right), U_{k}\left(\sigma_{k}\right)\right) \rightarrow\left(u_{\infty}(\sigma), U_{\infty}(\sigma)\right) \quad \text { weakly in } \mathbb{H} \text {. }
$$

By the minimizing property of $\sigma_{k}^{*}$ to the functional $J_{k}$ over $\mathscr{A}_{k}$, there holds $J_{k}\left(\sigma_{k}^{*}\right) \leqslant J_{k}\left(\sigma_{k}\right)$. Consequently,

$$
J_{\infty}\left(\sigma_{\infty}^{*}\right) \leqslant \liminf _{m \rightarrow \infty} J_{k_{m}}\left(\sigma_{k_{m}}^{*}\right) \leqslant \limsup _{k \rightarrow \infty} J_{k}\left(\sigma_{k}^{*}\right) \leqslant \limsup _{k \rightarrow \infty} J_{k}\left(\sigma_{k}\right)=J_{\infty}(\sigma) \quad \forall \sigma \in \mathscr{A}_{\infty} .
$$

Further, by taking $\sigma=\sigma_{\infty}^{*}$, we derive $\lim _{m \rightarrow \infty} J_{k_{m}}\left(\sigma_{k_{m}}^{*}\right)=J_{\infty}\left(\sigma_{\infty}^{*}\right)$, and thus $\lim _{m \rightarrow \infty}\left\|\nabla \sigma_{k_{m}}^{*}\right\|_{L^{2}(\Omega)}^{2}=\left\|\nabla \sigma_{\infty}^{*}\right\|_{L^{2}(\Omega)}^{2}$. This and the weak convergence of $\sigma_{k_{m}}^{*}$ in $H^{1}(\Omega)$ shows the first assertion. It remains to show the convergence of $\left\{u_{k_{m}}^{*}\right\}$ in $H^{1}(\Omega)$, which follows directly from the identity $\left\|\nabla\left(u_{k_{m}}^{*}-u_{\infty}^{*}\right)\right\|_{L^{2}(\Omega)} \rightarrow 0$. Using the discrete problem (3.1) over $\mathscr{T}_{k_{m}}$, the convergence of $\left\{U_{k_{m}}^{*}\right\}$ and the limiting problem (4.2) imply

$$
a\left(\sigma_{k_{m}},\left(u_{k_{m}}^{*}, U_{k_{m}}^{*}\right),\left(u_{k_{m}}^{*}, U_{k_{m}}^{*}\right)\right)=\left\langle I, U_{k_{m}}^{*}\right\rangle \rightarrow\left\langle I, U_{\infty}^{*}\right\rangle=a\left(\sigma_{\infty},\left(u_{\infty}^{*}, U_{\infty}^{*}\right),\left(u_{\infty}^{*}, U_{\infty}^{*}\right)\right),
$$

By the compact embedding from the trace $H^{1 / 2}(\Gamma)$ of $H^{1}(\Omega)$ into $L^{2}(\Gamma)$, the sequence $\left\{u_{k_{m}}^{*}\right\}$ converges to $u_{\infty}^{*}$ in $L^{2}(\Gamma)$, and the convergence of $\left\{U_{k_{m}}^{*}\right\}$ yield $\left(\sigma_{k_{m}}^{*} \nabla u_{k_{m}}^{*}, \nabla u_{k_{m}}^{*}\right) \rightarrow\left(\sigma_{\infty}^{*} \nabla u_{\infty}^{*}, \nabla u_{\infty}^{*}\right)$. By the identity

$$
\left\|\sqrt{\sigma_{k_{m}}^{*}} \nabla\left(u_{k_{m}}^{*}-u_{\infty}^{*}\right)\right\|_{L^{2}(\Omega)}^{2}=\left\|\sqrt{\sigma_{k_{m}}^{*}} \nabla u_{k_{m}}^{*}\right\|_{L^{2}(\Omega)}^{2}-2\left(\sigma_{k_{m}}^{*} \nabla u_{k_{m}}^{*}, \nabla u_{\infty}^{*}\right)+\left\|\sqrt{\sigma_{k_{m}}^{*}} \nabla u_{\infty}^{*}\right\|_{L^{2}(\Omega)}^{2}
$$

and the triangle inequality, we deduce

$$
\begin{aligned}
\left\|\nabla\left(u_{k_{m}}^{*}-u_{\infty}^{*}\right)\right\|_{L^{2}(\Omega)}^{2} \leqslant & c\left(\left|\left(\sigma_{k_{m}}^{*} \nabla u_{k_{m}}^{*}, \nabla u_{k_{m}}^{*}\right)-\left(\sigma_{\infty}^{*} \nabla u_{\infty}^{*}, \nabla u_{\infty}^{*}\right)\right|+\left|\left(\sigma_{k_{m}}^{*}-\sigma_{\infty}^{*},\left|\nabla u_{\infty}^{*}\right|^{2}\right)\right|\right. \\
& \left.+\left|\left(\sigma_{k_{m}}^{*} \nabla u_{k_{m}}^{*}-\sigma_{\infty}^{*} \nabla u_{\infty}^{*}, \nabla u_{\infty}^{*}\right)\right|\right):=\mathrm{I}+\mathrm{II}+\mathrm{III}
\end{aligned}
$$

The second term II tends to zero by the pointwise convergence of the sequence $\left\{\sigma_{k_{m}}^{*}\right\}$ and Lebesgue's dominated convergence theorem (Evans \& Gariepy, 1992, pp. 20). For the third term III, there holds

$$
\begin{aligned}
\mathrm{III} & \leqslant\left|\left(\left(\sigma_{k_{m}}^{*}-\sigma_{\infty}^{*}\right) \nabla u_{k_{m}}^{*}, \nabla u_{\infty}^{*}\right)\right|+\left|\left(\sigma_{\infty}^{*} \nabla\left(u_{k_{m}}^{*}-u_{\infty}^{*}\right), \nabla u_{\infty}^{*}\right)\right| \\
& \leqslant\left\|\nabla u_{k_{m}}^{*}\right\|_{L^{2}(\Omega)}\left\|\left(\sigma_{k_{m}}^{*}-\sigma_{\infty}^{*}\right) \nabla u_{\infty}^{*}\right\|_{L^{2}(\Omega)}+\left|\left(\sigma_{\infty}^{*} \nabla\left(u_{k_{m}}^{*}-u_{\infty}^{*}\right), \nabla u_{\infty}^{*}\right)\right| \rightarrow 0
\end{aligned}
$$

by the weak convergence of $\left\{u_{k_{m}}^{*}\right\}$ in $H^{1}(\Omega)$ and the pointwise convergence of $\left\{\sigma_{k_{m}}^{*}\right\}$. The preceding three estimates together complete the proof of the theorem.

Next we turn to the optimality system of problem (4.1). Like in the continuous case, the optimality condition for the minimizer $\left(\sigma_{\infty}^{*}, u_{\infty}^{*}, U_{\infty}^{*}\right)$ and the adjoint solution $\left(p_{\infty}^{*}, P_{\infty}^{*}\right) \in \mathbb{H}_{\infty}$ is given by

$$
\begin{aligned}
a\left(\sigma_{\infty}^{*},\left(u_{\infty}^{*}, U_{\infty}^{*}\right),(v, V)\right)=\langle I, V\rangle \quad \forall(v, V) \in \mathbb{H}_{\infty}, \\
a\left(\sigma_{\infty}^{*},\left(p_{\infty}^{*}, P_{\infty}^{*}\right),(v, V)\right)=\left\langle U_{\infty}^{*}-U^{\delta}, V\right\rangle \quad \forall(v, V) \in \mathbb{H}_{\infty}, \\
\alpha\left(\nabla \sigma_{\infty}^{*}, \nabla\left(\mu-\sigma_{\infty}^{*}\right)\right)-\left(\nabla u_{\infty}^{*}, \nabla p_{\infty}^{*}\left(\mu-\sigma_{\infty}^{*}\right)\right) \geqslant 0 \quad \forall \mu \in \mathscr{A}_{\infty} .
\end{aligned}
$$

The next result shows the convergence of the sequence of adjoint solutions. 
THEOREM 4.5 Under the condition of Theorem 4.4 , the subsequence of adjoint solutions $\left\{\left(p_{k_{m}}^{*}, P_{k_{m}}^{*}\right)\right\}$ generated by Algorithm 1 converges to the solution $\left(p_{\infty}^{*}, P_{\infty}^{*}\right)$ to the limiting adjoint problem in (4.5):

$$
\lim _{m \rightarrow \infty}\left\|\left(p_{k_{m}}^{*}-p_{\infty}^{*}, P_{k_{m}}^{*}-P_{\infty}^{*}\right)\right\|_{\mathbb{H}, *}=0
$$

Proof. The discrete version of the limiting adjoint problem (4.5) reads: find $\left(\widetilde{p}_{k_{m}}, \widetilde{P}_{k_{m}}\right) \in \mathbb{H}_{k_{m}}$ such that

$$
a\left(\sigma_{\infty}^{*},\left(\widetilde{p}_{k_{m}}, \widetilde{P}_{k_{m}}\right),(v, V)\right)=\left\langle U_{\infty}^{*}-U^{\delta}, V\right\rangle \quad \forall(v, V) \in \mathbb{H}_{k_{m}} .
$$

By Cea's lemma and the construction of the space $\mathbb{H}_{\infty}$, we deduce

$$
\left\|\left(p_{\infty}^{*}-\widetilde{p}_{k_{m}}, P_{\infty}^{*}-\widetilde{P}_{k_{m}}\right)\right\|_{\mathbb{H}, *} \leqslant c \inf _{(v, V) \in \mathbb{H}_{k_{m}}}\left\|\left(p_{\infty}^{*}-v, P_{\infty}^{*}-V\right)\right\|_{\mathbb{H}, *} \rightarrow 0 .
$$

By taking $\left(v_{k_{m}}, V_{k_{m}}\right)=\left(\widetilde{p}_{k_{m}}-p_{k_{m}}^{*}, \widetilde{P}_{k_{m}}-P_{k_{m}}^{*}\right)$ in the second equation of (3.3) and $(v, V)=\left(\widetilde{p}_{k_{m}}-p_{k_{m}}^{*}, \widetilde{P}_{k_{m}}-\right.$ $\left.P_{k_{m}}^{*}\right)$ in (4.6), we obtain

$$
\begin{aligned}
\left\|\sqrt{\sigma_{k_{m}}} \nabla\left(\widetilde{p}_{k_{m}}-p_{k_{m}}^{*}\right)\right\|_{L^{2}(\Omega)}^{2}+\sum_{l=1}^{L} z_{l}^{-1}\left\|\widetilde{p}_{k_{m}}-p_{k_{m}}^{*}-\widetilde{P}_{k_{m}, l}+P_{k_{m}, l}^{*}\right\|_{L^{2}\left(e_{l}\right)}^{2} \\
=\left\langle U_{\infty}^{*}-U_{k_{m}}^{*}, \widetilde{P}_{k_{m}}-P_{k_{m}}^{*}\right\rangle+\left(\left(\sigma_{k_{m}}^{*}-\sigma_{\infty}^{*}\right) \nabla\left(\widetilde{p}_{k_{m}}-p_{\infty}^{*}\right), \nabla\left(\widetilde{p}_{k_{m}}-p_{k_{m}}^{*}\right)\right) \\
\quad+\left(\left(\sigma_{k_{m}}^{*}-\sigma_{\infty}^{*}\right) \nabla p_{\infty}^{*}, \nabla\left(\widetilde{p}_{k_{m}}-p_{k_{m}}^{*}\right)\right):=\mathrm{I}+\mathrm{II}+\mathrm{III} .
\end{aligned}
$$

The Cauchy-Schwarz inequality and the box constraints on $\sigma_{k_{m}}^{*}$ and $\sigma_{\infty}^{*}$ give

$$
\begin{aligned}
|\mathrm{I}| & \leqslant\left\|U_{\infty}^{*}-U_{k_{m}}^{*}\right\|_{\mathbb{R}^{L}}\left\|\widetilde{P}_{k_{m}}-P_{k_{m}}^{*}\right\|_{\mathbb{R}^{L}}, \\
|\mathrm{II}| & \leqslant c\left\|\nabla\left(\widetilde{p}_{k_{m}}-p_{\infty}^{*}\right)\right\|_{L^{2}(\Omega)}\left\|\nabla\left(\widetilde{p}_{k_{m}}-p_{k_{m}}^{*}\right)\right\|_{L^{2}(\Omega)}, \\
|\mathrm{III}| & \leqslant\left\|\left(\sigma_{k_{m}}^{*}-\sigma_{\infty}^{*}\right) \nabla p_{\infty}^{*}\right\|_{L^{2}(\Omega)}\left\|\nabla\left(\widetilde{p}_{k_{m}}-p_{k_{m}}^{*}\right)\right\|_{L^{2}(\Omega)},
\end{aligned}
$$

which, together with Lemma 2.1, implies

$$
\left\|\left(\widetilde{p}_{k_{m}}-p_{k_{m}}^{*}, \widetilde{P}_{k_{m}}-P_{k_{m}}^{*}\right)\right\|_{\mathbb{H}, *} \leqslant c\left(\left\|U_{\infty}^{*}-U_{k_{m}}^{*}\right\|_{\mathbb{R}^{L}}+\left\|\nabla\left(\widetilde{p}_{k_{m}}-p_{\infty}^{*}\right)\right\|_{L^{2}(\Omega)}+\left\|\left(\sigma_{k_{m}}^{*}-\sigma_{\infty}^{*}\right) \nabla p_{\infty}^{*}\right\|_{L^{2}(\Omega)}\right) .
$$

Thanks to the convergence of $\left\{U_{k_{m}}^{*}\right\}$, the pointwise convergence of $\left\{\sigma_{k_{m}}^{*}\right\}$ in Theorem 4.4 and (4.7), the right-hand side tends to zero. Now the desired assertion follows from the triangle inequality and (4.7).

\subsection{Convergence of AFEM}

Now we establish the main theoretical result of this work: the sequence of discrete solutions generated by Algorithm 1 contains a convergent subsequence $\left\{\left(\sigma_{k_{m}}^{*}, u_{k_{m}}^{*}, U_{k_{m}}^{*}, p_{k_{m}}^{*}, P_{k_{m}}^{*}\right)\right\}$, and the limit satisfies the optimality system (2.5). By Theorems 4.4 and 4.5 , it suffices to show that the limit $\left\{\left(\sigma_{\infty}^{*}, u_{\infty}^{*}, U_{\infty}^{*}, p_{\infty}^{*}, P_{\infty}^{*}\right)\right\}$ solves (2.5). Our arguments begin with the observation that the maximal error indicator over marked elements has a vanishing limit, cf. Lemma 4.6. Then we show that the sequences of residuals with respect to $\left(u_{k_{m}}^{*}, U_{k_{m}}^{*}\right)$ and $\left(p_{k_{m}}^{*}, P_{k_{m}}^{*}\right)$ converge to zero weakly in Lemma 4.7. This and Theorems 4.4 and 4.5 verify the first two lines in (2.5) in Lemma 4.8, and the variational inequality in Lemma 4.9.

First we show that the maximal error indicator over the marked elements has a vanishing limit. 
LEMma 4.6 Let $\left\{\mathscr{T}_{k}, \mathscr{A}_{k} \times \mathbb{H}_{k},\left(\sigma_{k}^{*}, u_{k}^{*}, U_{k}^{*}, p_{k}^{*}, P_{k}^{*}\right)\right\}$ be the sequence of meshes, discrete admissible sets, finite element spaces and discrete solutions generated by Algorithm 1 and $\mathscr{M}_{k}$ the set of marked elements by (3.6). Then for each convergent subsequence $\left\{\left(\sigma_{k_{m}}^{*}, u_{k_{m}}^{*}, U_{k_{m}}^{*}, p_{k_{m}}^{*}, P_{k_{m}}^{*}\right)\right\}$, there holds

$$
\lim _{m \rightarrow \infty} \max _{T \in \mathscr{M}_{k_{m}}} \eta_{k_{m}}\left(\sigma_{k_{m}}^{*}, u_{k_{m}}^{*}, U_{k_{m}}^{*}, p_{k_{m}}^{*}, P_{k_{m}}^{*}, T\right)=0
$$

Proof. We denote by $\widetilde{T}$ the element with the largest error indicator in $\mathscr{M}_{k_{m}}$. Since the set $D_{\widetilde{T}} \subset \Omega_{k_{m}}^{0}$, it follows from Lemma 3.4 that

$$
\left|D_{\widetilde{T}}\right| \leqslant c\left\|h_{k_{m}}\right\|_{L^{\infty}\left(\Omega_{k_{m}}^{0}\right)}^{d} \rightarrow 0, \quad\left|\partial \widetilde{T} \cap e_{l}\right| \leqslant c\left\|h_{k_{m}}\right\|_{L^{\infty}\left(\Omega_{k_{m}}^{0}\right)}^{d-1} \rightarrow 0 \quad \text { as } m \rightarrow \infty .
$$

By Lemma 3.5, the local quasi-uniformity of $\mathscr{T}_{k_{m}}$, inverse estimates, trace theorem (Evans \& Gariepy, 1992, pp. 133) and the triangle inequality, we have

$$
\begin{aligned}
& \eta_{k_{m}, 1}^{2}\left(\sigma_{k_{m}}^{*}, u_{k_{m}}^{*}, U_{k_{m}}^{*}, \widetilde{T}\right) \leqslant c\left(\left\|\nabla u_{k_{m}}^{*}\right\|_{L^{2}\left(D_{\widetilde{T}}\right)}^{2}+h_{\widetilde{T}}\left\|u_{k_{m}, l}^{*}-U_{k_{m}, l}^{*}\right\|_{L^{2}\left(\partial \widetilde{T} \cap e_{l}\right)}^{2}\right) \\
& \leqslant c\left(\left\|\left(u_{k_{m}}^{*}-u_{\infty}^{*}, U_{k_{m}}^{*}-U_{\infty}^{*}\right)\right\|_{\mathbb{H}, *}^{2}+\left\|\nabla u_{\infty}^{*}\right\|_{L^{2}\left(D_{\widetilde{T}}\right)}^{2}+\left\|u_{\infty, l}^{*}-U_{\infty, l}^{*}\right\|_{L^{2}\left(\partial \widetilde{T} \cap e_{l}\right)}^{2}\right), \\
& \eta_{k_{m}, 2}^{2}\left(\sigma_{k_{m}}^{*}, p_{k_{m}}^{*}, P_{k_{m}}^{*}, \widetilde{T}\right) \leqslant c\left(\left\|\nabla p_{k_{m}}^{*}\right\|_{L^{2}\left(D_{\widetilde{T}}\right)}^{2}+h_{\widetilde{T}}\left\|p_{k_{m}, l}^{*}-P_{k_{m}, l}^{*}\right\|_{L^{2}\left(\partial \widetilde{T} \cap e_{l}\right)}^{2}\right) \\
& \leqslant c\left(\left\|\left(p_{k_{m}}^{*}-p_{\infty}^{*}, P_{k_{m}}^{*}-P_{\infty}^{*}\right)\right\|_{\mathbb{H}_{1}, *}^{2}+\left\|\nabla p_{\infty}^{*}\right\|_{L^{2}\left(D_{\widetilde{T}}\right)}^{2}+\left\|p_{\infty, l}^{*}-P_{\infty, l}^{*}\right\|_{L^{2}\left(\partial \widetilde{T} \cap e_{l}\right)}^{2}\right), \\
& \eta_{k_{m}, 3}^{2}\left(\sigma_{k_{m}}^{*}, u_{k_{m}}^{*}, p_{k_{m}}^{*}, \widetilde{T}\right) \leqslant c\left(h_{\widetilde{T}}^{4-d}\left\|\nabla u_{k_{m}}^{*}\right\|_{L^{2}(\widetilde{T})}^{2}\left\|\nabla p_{k_{m}}^{*}\right\|_{L^{2}(\widetilde{T})}^{2}+h_{\widetilde{T}}^{2}\left\|\nabla \sigma_{k_{m}}^{*}\right\|_{L^{2}\left(D_{\widetilde{T}}\right)}^{2}\right) \\
& \leqslant c\left|D_{\widetilde{T}}\right|^{4 / d-1}\left(\left(\left\|\nabla\left(u_{k_{m}}^{*}-u_{\infty}^{*}\right)\right\|_{L^{2}(\widetilde{T})}^{2}+\left\|\nabla u_{\infty}^{*}\right\|_{L^{2}(\widetilde{T})}^{2}\right)\left(\left\|\nabla\left(p_{k_{m}}^{*}-p_{\infty}^{*}\right)\right\|_{L^{2}(\widetilde{T})}^{2}+\left\|\nabla p_{\infty}^{*}\right\|_{L^{2}(\widetilde{T})}^{2}\right)\right. \\
&\left.+\left(\left\|\nabla\left(\sigma_{k_{m}}^{*}-\sigma_{\infty}^{*}\right)\right\|_{L^{2}\left(D_{\widetilde{T}}\right)}^{2}+\left\|\nabla \sigma_{\infty}^{*}\right\|_{L^{2}\left(D_{\widetilde{T}}\right)}^{2}\right)\right) .
\end{aligned}
$$

The desired result follows from Theorems 4.4 and 4.5, (4.8), and the absolute continuity of the norms $\|\cdot\|_{L^{2}(\Omega)}$ and $\|\cdot\|_{L^{2}(\Gamma)}$ with respect to the Lebesgue measure.

Now we define two residuals with respect to $\left(u_{k}^{*}, U_{k}^{*}\right)$ and $\left(p_{k}^{*}, P_{k}^{*}\right)$ as

$$
\begin{aligned}
& \left\langle\mathscr{R}\left(u_{k}^{*}, U_{k}^{*}\right),(v, V)\right\rangle:=a\left(\sigma_{k}^{*},\left(u_{k}^{*}, U_{k}^{*}\right),(v, V)\right)-\langle I, V\rangle \quad \forall(v, V) \in \mathbb{H}, \\
& \left\langle\mathscr{R}\left(p_{k}^{*}, P_{k}^{*}\right),(v, V)\right\rangle:=a\left(\sigma_{k}^{*},\left(p_{k}^{*}, P_{k}^{*}\right),(v, V)\right)-\left\langle U_{k}^{*}-U^{\delta}, V\right\rangle \quad \forall(v, V) \in \mathbb{H} .
\end{aligned}
$$

By definition, we have the following Galerkin orthogonality

$$
\begin{array}{ll}
\left\langle\mathscr{R}\left(p_{k}^{*}, P_{k}^{*}\right),(v, V)\right\rangle=0 & \forall(v, V) \in \mathbb{H}_{k}, \\
\left\langle\mathscr{R}\left(p_{k}^{*}, P_{k}^{*}\right),(v, V)\right\rangle=0 & \forall(v, V) \in \mathbb{H}_{k} .
\end{array}
$$

To relate the limit $\left\{\left(\sigma_{\infty}^{*}, u_{\infty}^{*}, U_{\infty}^{*}, p_{\infty}^{*}, P_{\infty}^{*}\right)\right\}$ to the optimality system (2.5), we exploit the marking assumption (3.6) in Algorithm 1. The next result gives the weak convergence of the residuals to zero.

LEMmA 4.7 For the convergent subsequence $\left\{\left(\sigma_{k_{m}}^{*}, u_{k_{m}}^{*}, U_{k_{m}}^{*}, p_{k_{m}}^{*}, P_{k_{m}}^{*}\right)\right\}$ given in Theorems 4.4 and 4.5, there hold

$$
\begin{aligned}
\lim _{m \rightarrow \infty}\left\langle\mathscr{R}\left(u_{k_{m}}^{*}, U_{k_{m}}^{*}\right),(v, V)\right\rangle=0 & \forall(v, V) \in \mathbb{H}, \\
\lim _{m \rightarrow \infty}\left\langle\mathscr{R}\left(p_{k_{m}}^{*}, P_{k_{m}}^{*}\right),(v, V)\right\rangle=0 & \forall(v, V) \in \mathbb{H} .
\end{aligned}
$$


Proof. We only prove the first assertion since the second follows analogously, and relabel the index $k_{m}$ by $k$. Let $I_{k}$ and $I_{k}^{s z}$ be the Lagrange and Scott-Zhang interpolation operators respectively associated with $V_{k}$. Then by (4.9), elementwise integration by parts and Lemma 3.6, we deduce for $k>l$ and any $(\psi, V) \in C^{\infty}(\bar{\Omega}) \times \mathbb{R}_{\diamond}^{L}$

$$
\begin{aligned}
\left|\left\langle\mathscr{R}\left(u_{k}^{*}, U_{k}^{*}\right),(\psi, V)\right\rangle\right| & =\left|\left\langle\mathscr{R}\left(u_{k}^{*}, U_{k}^{*}\right),\left(\psi-I_{k} \psi, 0\right)\right\rangle\right|=\left|\left\langle\mathscr{R}\left(u_{k}^{*}, U_{k}^{*}\right),\left(w-I_{k}^{s z} w, 0\right)\right\rangle\right| \\
& =\left|\left(\sigma_{k}^{*} \nabla u_{k}^{*}, \nabla\left(w-I_{k}^{s z} w\right)\right)+\sum_{l=1}^{L} z_{l}^{-1}\left(\left(u_{k}^{*}-U_{k, l}^{*}\right),\left(w-I_{k}^{s z} w\right)\right)_{L^{2}\left(e_{l}\right)}\right| \\
& \leqslant c \sum_{T \in \mathscr{T}_{k}} \eta_{k, 1}\left(\sigma_{k}^{*}, u_{k}^{*}, U_{k}^{*}, T\right)\|w\|_{H^{1}\left(D_{T}\right)} \\
& =c\left(\sum_{T \in \mathscr{T}_{k} \backslash \mathscr{T}_{l}^{+}} \eta_{k, 1}\left(\sigma_{k}^{*}, u_{k}^{*}, U_{k}^{*}, T\right)\|w\|_{H^{1}\left(D_{T}\right)}+\sum_{T \in \mathscr{T}_{l}^{+}} \eta_{k, 1}\left(\sigma_{k}^{*}, u_{k}^{*}, U_{k}^{*}, T\right)\|w\|_{H^{1}\left(D_{T}\right)}\right) .
\end{aligned}
$$

where $w=\psi-I_{k} \psi$. By appealing to Lemma 3.5 and (3.4), we deduce

$$
\left(\sum_{T \in \mathscr{T}_{k} \backslash \mathscr{T}_{l}^{+}} \eta_{k, 1}^{2}\left(\sigma_{k}^{*}, u_{k}^{*}, U_{k}^{*}, T\right)\right)^{1 / 2} \leqslant c
$$

and further by the error estimate of the interpolation operator $I_{k}$ from Lemma 3.6, we arrive at

$$
\left|\left\langle\mathscr{R}\left(u_{k}^{*}, U_{k}^{*}\right),(\psi, V)\right\rangle\right| \leqslant c_{1}\left\|h_{l}\right\|_{L^{\infty}\left(\Omega_{l}^{0}\right)}\|\psi\|_{H^{2}(\Omega)}+c_{2}\left(\sum_{T \in \mathscr{T}_{l}^{+}} \eta_{k, 1}^{2}\left(\sigma_{k}^{*}, u_{k}^{*}, U_{k}^{*}, T\right)\right)^{1 / 2}\|\psi\|_{H^{2}(\Omega)} .
$$

By Lemma 3.4, $c_{1}\left\|h_{l}\right\|_{L^{\infty}\left(\Omega_{l}^{0}\right)}\|\psi\|_{2} \rightarrow 0$ as $l \rightarrow \infty$. From $\mathscr{T}_{l}^{+} \subset \mathscr{T}_{k}^{+} \subset \mathscr{T}_{k} \subset \mathscr{M}_{k}$ for $k>l$ and the marking condition (3.6), we deduce

$$
\begin{aligned}
\left(\sum_{T \in \mathscr{T}_{l}^{+}} \eta_{k, 1}^{2}\left(\sigma_{k}^{*}, u_{k}^{*}, U_{k}^{*}, T\right)\right)^{1 / 2} & \leqslant \sqrt{\left|\mathscr{T}_{l}^{+}\right|} \max _{T \in \mathscr{T}_{l}^{+}} \eta_{k, 1}\left(\sigma_{k}^{*}, u_{k}^{*}, U_{k}^{*}, T\right) \leqslant \sqrt{\left|\mathscr{T}_{l}^{+}\right|} \max _{T \in \mathscr{T}_{k}^{+}} \eta_{k, 1}\left(\sigma_{k}^{*}, u_{k}^{*}, U_{k}^{*}, T\right) \\
& \leqslant \sqrt{\left|\mathscr{T}_{l}^{+}\right|} \max _{T \in \mathscr{M}_{k}} \eta_{k}\left(\sigma_{k}^{*}, u_{k}^{*}, U_{k}^{*}, p_{k}^{*}, P_{k}^{*}, T\right)
\end{aligned}
$$

Now Lemma 4.6 implies that for any fixed large $l_{1}$, we can choose some $k_{1}>l_{1}$ such that

$$
c_{2}\left(\sum_{T \in \mathscr{T}_{l}^{+}} \eta_{k, 1}^{2}\left(\sigma_{k}^{*}, u_{k}^{*}, U_{k}^{*}, T\right)\right)^{1 / 2}\|\psi\|_{2}<\varepsilon
$$

for any positive small number $\varepsilon$ and $k>k_{1}$. Thus, we arrive at

$$
\lim _{m \rightarrow \infty}\left\langle\mathscr{R}\left(u_{k_{m}}^{*}, U_{k_{m}}^{*}\right),(v, V)\right\rangle=0 \quad \forall(v, V) \in C^{\infty}(\bar{\Omega}) \times \mathbb{R}_{\diamond}^{L},
$$

which, together with the density of $C^{\infty}(\bar{\Omega})$ in $H^{1}(\Omega)$, gives the desired assertion.

Next we show that the limit $\left(\sigma_{\infty}^{*}, u_{\infty}^{*}, U_{\infty}^{*}, p_{\infty}^{*}, P_{\infty}^{*}\right)$ actually solves the variational equations in (2.5).

LEMMA 4.8 The solution to problem (4.5) solves the two variational equations in (2.5), i.e.,

$$
\begin{aligned}
& a\left(\sigma_{\infty}^{*},\left(u_{\infty}^{*}, U_{\infty}^{*}\right),(v, V)\right)=\langle I, V\rangle \quad \forall(v, V) \in \mathbb{H}, \\
& a\left(\sigma_{\infty}^{*},\left(p_{\infty}^{*}, P_{\infty}^{*}\right),(v, V)\right)=\left\langle U_{\infty}^{*}-U^{\delta}, V\right\rangle \quad \forall(v, V) \in \mathbb{H} .
\end{aligned}
$$


Proof. We prove only the first assertion, since the proof of the second is analogous. Given the convergent subsequence $\left\{\left(\sigma_{k_{m}}^{*}, u_{k_{m}}^{*}, U_{k_{m}}^{*}, p_{k_{m}}^{*}, P_{k_{m}}^{*}\right)\right\}$ in Theorems 4.4 and 4.5 , for any $(v, V) \in \mathbb{H}$, there holds

$$
\begin{aligned}
\mid a\left(\sigma_{\infty}^{*},\left(u_{\infty}^{*}, U_{\infty}^{*}\right),(v, V)\right)- & \langle I, V\rangle\left|\leqslant \sum_{l=1}^{L} z_{l}^{-1}\right|\left(u_{\infty}^{*}-U_{\infty, l}^{*}-u_{k_{m}}^{*}+U_{k_{m}, l}^{*}, v-V_{l}\right)_{L^{2}\left(e_{l}\right)} \mid \\
& +\left|\left(\left(\sigma_{\infty}^{*} \nabla u_{\infty}^{*}-\sigma_{k_{m}}^{*} \nabla u_{k_{m}}^{*}\right), \nabla v\right)_{L^{2}(\Omega)}\right|+\left|\left\langle\mathscr{R}\left(u_{k_{m}}^{*}, U_{k_{m}}^{*}\right),(v, V)\right\rangle\right| .
\end{aligned}
$$

In view of Theorem 4.4 and Lemma 4.7, the first and third terms tend to zero. For the second term,

$$
\begin{aligned}
\left|\left(\left(\sigma_{\infty}^{*} \nabla u_{\infty}^{*}-\sigma_{k_{m}}^{*} u_{k_{m}}^{*}\right), \nabla v\right)\right| & \leqslant\left|\left(\sigma_{\infty}^{*} \nabla\left(u_{\infty}^{*}-u_{k_{m}}^{*}\right), \nabla v\right)\right|+\left|\left(\left(\sigma_{\infty}^{*}-\sigma_{k_{m}}^{*}\right) \nabla u_{k_{m}}^{*}, \nabla v\right)\right| \\
& \leqslant\left|\left(\sigma_{\infty}^{*} \nabla\left(u_{\infty}^{*}-u_{k_{m}}^{*}\right), \nabla v\right)\right|+\left\|\nabla u_{k_{m}}^{*}\right\|_{L^{2}(\Omega)}\left\|\left(\sigma_{\infty}^{*}-\sigma_{k_{m}}^{*}\right) \nabla v\right\|_{L^{2}(\Omega)} \rightarrow 0,
\end{aligned}
$$

by the convergence of $\left\{u_{k_{m}}^{*}\right\}$, and the pointwise convergence of $\left\{\sigma_{k_{m}}^{*}\right\}$ in Theorem 4.4 and Lebesgue's dominated convergence theorem (Evans \& Gariepy, 1992, pp. 20).

Now we turn to the variational inequality in (2.5). We resort again to a density argument: we first show the assertion over a smooth subset, and then extend it to $\mathscr{A}$ by a density argument.

Lemma 4.9 The solution to the variational inequality of problem (4.5) satisfies

$$
\alpha\left(\nabla \sigma_{\infty}^{*}, \nabla\left(\mu-\sigma_{\infty}^{*}\right)\right)-\left(\nabla u_{\infty}^{*}, \nabla p_{\infty}^{*}\left(\mu-\sigma_{\infty}^{*}\right)\right) \geqslant 0 \quad \forall \mu \in \mathscr{A} .
$$

Proof. Like before, we relabel the index $k_{m}$ by $k$, and let $I_{k}$ be the Lagrange interpolation operator associated with $V_{k}$. Then for any $\mu \in \widetilde{\mathscr{A}}:=\mathscr{A} \cap C^{\infty}(\bar{\Omega}), I_{k} \mu \in \mathscr{A}_{k}$ and the discrete variational inequality in (3.3) yields

$$
\begin{aligned}
& \alpha\left(\nabla \sigma_{k}^{*}, \nabla\left(\mu-\sigma_{k}^{*}\right)\right)-\left(\left(\mu-\sigma_{k}^{*}\right) \nabla u_{k}^{*}, \nabla p_{k}^{*}\right) \\
= & \alpha\left(\nabla \sigma_{k}^{*}, \nabla\left(\mu-I_{k} \mu\right)\right)-\left(\left(\mu-I_{k} \mu\right) \nabla u_{k}^{*}, \nabla p_{k}^{*}\right) \\
& +\alpha\left(\nabla \sigma_{k}^{*}, \nabla\left(I_{k} \mu-\sigma_{k}^{*}\right)\right)-\left(\left(I_{k} \mu-\sigma_{k}^{*}\right) \nabla u_{k}^{*}, \nabla p_{k}^{*}\right) \\
\geqslant & \alpha\left(\nabla \sigma_{k}^{*}, \nabla\left(\mu-I_{k} \mu\right)\right)-\left(\left(\mu-I_{k} \mu\right) \nabla u_{k}^{*}, \nabla p_{k}^{*}\right) .
\end{aligned}
$$

Using elementwise integration by parts, the definition of $\eta_{k, 3}$ and error estimates for $I_{k}$, cf. Lemma 3.6, we deduce that for $k>l$, there holds

$$
\begin{aligned}
\left|\alpha\left(\nabla \sigma_{k}^{*}, \nabla\left(\mu-I_{k} \mu\right)\right)-\left(\left(\mu-I_{k} \mu\right) \nabla u_{k}^{*}, \nabla p_{k}^{*}\right)\right| \leqslant c \sum_{T \in \mathscr{T}_{k}} \eta_{k, 3}\left(\sigma_{k}^{*}, u_{k}^{*}, p_{k}^{*}, T\right)\|\mu\|_{H^{2}(T)} \\
\leqslant c_{3}\left(\left(\sum_{T \in \mathscr{T}_{k} \backslash \mathscr{T}_{l}^{+}} \eta_{k, 3}^{2}\left(\sigma_{k}^{*}, u_{k}^{*}, p_{k}^{*}, T\right)\right)^{1 / 2}+\left(\sum_{T \in \mathscr{T}_{l}^{+}} \eta_{k, 3}^{2}\left(\sigma_{k}^{*}, u_{k}^{*}, p_{k}^{*}, T\right)\right)^{1 / 2}\right)\|\mu\|_{H^{2}(\Omega)} .
\end{aligned}
$$

The Lemma 3.5, (3.4), Theorem 4.4 and Lemma 3.4 give

$$
\begin{aligned}
\sum_{T \in \mathscr{T}_{k} \backslash \mathscr{T}_{l}^{+}} \eta_{k, 3}^{2}\left(\sigma_{k}^{*}, u_{k}^{*}, p_{k}^{*}, T\right) & \leqslant c\left(\left\|h_{l}\right\|_{L^{\infty}\left(\Omega_{0}^{l}\right)}^{4-d}\left\|\nabla p_{k}\right\|_{L^{2}(\Omega)}^{2} \sum_{T \in \mathscr{T}_{k} \backslash \mathscr{T}_{l}^{+}}\left\|\nabla u_{k}^{*}\right\|_{L^{2}(T)}^{2}+\left\|h_{l}\right\|_{L^{\infty}\left(\Omega_{0}^{l}\right)}^{2}\left\|\nabla \sigma_{k}^{*}\right\|_{L^{2}(\Omega)}^{2}\right) \\
& \leqslant c\left(\left\|h_{l}\right\|_{L^{\infty}\left(\Omega_{0}^{l}\right)}^{4-d}+\left\|h_{l}\right\|_{L^{\infty}\left(\Omega_{0}^{l}\right)}^{2}\right) \leqslant c\left\|h_{l}\right\|_{L^{\infty}\left(\Omega_{0}^{l}\right)}^{4-d} \rightarrow 0 .
\end{aligned}
$$

Upon noting the inclusion $\mathscr{T}_{l}^{+} \subset \mathscr{T}_{k}$ for $k>l$, we deduce from the marking condition (3.6)

$\left(\sum_{T \in \mathscr{T}_{l}^{+}} \eta_{k, 3}^{2}\left(\sigma_{k}^{*}, u_{k}^{*}, p_{k}^{*}, T\right)\right)^{1 / 2} \leqslant \sqrt{\left|\mathscr{T}_{l}^{+}\right|} \max _{T \in \mathscr{T}_{l}^{+}} \eta_{k, 3}\left(\sigma_{k}^{*}, u_{k}^{*}, p_{k}^{*}, T\right) \leqslant \sqrt{\left|\mathscr{T}_{l}^{+}\right|} \max _{T \in \mathscr{M}_{k}} \eta_{k}\left(\sigma_{k}^{*}, u_{k}^{*}, U_{k}^{*}, p_{k}^{*}, P_{k}^{*}, T\right)$. 
Appealing again to Lemma 4.6, we can choose $k_{2}>l_{2}$ for some large fixed $l_{2}$ such that when $k>k_{2}$ $c_{3}\left(\sum_{T \in \mathscr{T}_{1}^{+}} \eta_{k, 3}^{2}\left(\sigma_{k}^{*}, u_{k}^{*}, p_{k}^{*}, T\right)\right)^{1 / 2}\|\mu\|_{H^{2}(\Omega)}$ is smaller than any given positive number. Hence

$$
\left(\alpha \nabla \sigma_{k}^{*}, \nabla\left(\mu-I_{k} \mu\right)\right)-\left(\nabla u_{k}^{*}, \nabla p_{k}^{*}\left(\mu-I_{k} \mu\right)\right) \rightarrow 0 \quad \forall \mu \in \widetilde{\mathscr{A}} .
$$

Using the $H^{1}(\Omega)$-convergence of $\left\{\sigma_{k}^{*}\right\}$ from Theorem 4.4, we have

$$
\left(\alpha \nabla \sigma_{k}^{*}, \nabla\left(\mu-\sigma_{k}^{*}\right)\right) \rightarrow\left(\alpha \nabla \sigma_{\infty}^{*}, \nabla\left(\mu-\sigma_{\infty}^{*}\right)\right) \quad \forall \mu \in \widetilde{\mathscr{A}} .
$$

The convergence of $\left\{p_{k}^{*}\right\}$ to $p_{\infty}^{*}$ in $H^{1}(\Omega)$ in Theorem 4.5, (3.4) and the box constraint in $\widetilde{\mathscr{A}}$ yield

$$
\left(\mu \nabla u_{k}^{*}, \nabla\left(p_{k}^{*}-p_{\infty}^{*}\right)\right) \leqslant c\left\|\nabla\left(p_{k}^{*}-p_{\infty}^{*}\right)\right\|_{L^{2}(\Omega)} \rightarrow 0,
$$

and this together with Theorem 4.4 implies

$$
\left(\mu \nabla u_{k}^{*}, \nabla p_{k}^{*}\right)=\left(\mu \nabla u_{k}^{*}, \nabla\left(p_{k}^{*}-p_{\infty}^{*}\right)\right)+\left(\mu \nabla u_{k}^{*}, \nabla p_{\infty}^{*}\right) \rightarrow\left(\mu \nabla u_{\infty}^{*}, \nabla p_{\infty}^{*}\right) \quad \forall \mu \in \widetilde{\mathscr{A}} .
$$

By elementary calculations, we derive

$$
\begin{aligned}
\left(\sigma_{k}^{*} \nabla u_{k}^{*}, \nabla p_{k}^{*}\right)-\left(\sigma_{\infty}^{*} \nabla u_{\infty}^{*}, \nabla p_{\infty}^{*}\right)= & \left(\sigma_{k}^{*} \nabla u_{k}^{*}, \nabla\left(p_{k}^{*}-p_{\infty}^{*}\right)\right)+\left(\left(\sigma_{k}^{*}-\sigma_{\infty}^{*}\right) \nabla u_{k}^{*}, \nabla p_{\infty}^{*}\right) \\
& +\left(\sigma_{\infty}^{*} \nabla\left(u_{k}^{*}-u_{\infty}^{*}\right), \nabla p_{\infty}^{*}\right) .
\end{aligned}
$$

Repeating the arguments for (4.13) yields that for the first and third terms there hold $\left(\sigma_{k}^{*} \nabla u_{k}^{*}, \nabla\left(p_{k}^{*}-p_{\infty}^{*}\right)\right) \rightarrow$ 0 and $\left(\sigma_{\infty}^{*} \nabla\left(u_{k}^{*}-u_{\infty}^{*}\right), \nabla p_{\infty}^{*}\right) \rightarrow 0$. The stability estimate (3.4), the pointwise convergence of $\left\{\sigma_{k}^{*}\right\}$ of Theorem 4.4 and Lebesgue's dominated convergence theorem (Evans \& Gariepy, 1992, pp. 20) show

$$
\left(\left(\sigma_{k}^{*}-\sigma_{\infty}^{*}\right) \nabla u_{k}^{*}, \nabla p_{\infty}^{*}\right) \leqslant c\left\|\left(\sigma_{k}^{*}-\sigma_{\infty}^{*}\right) \nabla p_{\infty}^{*}\right\|_{L^{2}(\Omega)} \rightarrow 0 .
$$

Hence

$$
\left(\sigma_{k}^{*} \nabla u_{k}^{*}, \nabla p_{k}^{*}\right) \rightarrow\left(\sigma_{\infty}^{*} \nabla u_{\infty}^{*}, \nabla p_{\infty}^{*}\right) .
$$

Now by passing both sides of (4.10) to the limit and combining (4.11)-(4.14), we obtain

$$
\alpha\left(\nabla \sigma_{\infty}^{*}, \nabla\left(\mu-\sigma_{\infty}^{*}\right)\right)_{L^{2}(\Omega)}-\left(\nabla u_{\infty}^{*}, \nabla p_{\infty}^{*}\left(\mu-\sigma_{\infty}^{*}\right)\right)_{L^{2}(\Omega)} \geqslant 0 \quad \forall \mu \in \widetilde{\mathscr{A}} .
$$

By means of the density of $C^{\infty}(\bar{\Omega})$ in $H^{1}(\Omega)$ and the construction via a standard mollifier (Evans \& Gariepy, 1992, pp. 122), for any $\mu \in \mathscr{A}$ there exists a sequence $\left\{\mu^{n}\right\} \subset \widetilde{\mathscr{A}}$ such that $\left\|\mu^{n}-\mu\right\|_{H^{1}(\Omega)} \rightarrow 0$ as $n \rightarrow \infty$. Then by Lebesgue's dominated convergence theorem (Evans \& Gariepy, 1992, pp. 20), we deduce

$$
\left(\alpha \nabla \sigma_{\infty}^{*}, \nabla \mu^{n}\right) \rightarrow\left(\alpha \nabla \sigma_{\infty}^{*}, \nabla \mu\right) \quad \text { and } \quad\left(\mu^{n} \nabla u_{\infty}^{*}, \nabla p_{\infty}^{*}\right) \rightarrow\left(\mu \nabla u_{\infty}^{*}, \nabla p_{\infty}^{*}\right)
$$

after possibly passing to a subsequence. The desired result follows from the preceding two estimates.

Finally, by combining preceding results, we obtain the main theoretical result: the sequence of solutions generated by the AFEM contains a subsequence converging to a solution of (2.5).

THEOREM 4.10 The sequence of discrete solutions $\left\{\left(\sigma_{k}^{*}, u_{k}^{*}, U_{k}^{*}, p_{k}^{*}, P_{k}^{*}\right)\right\}$ generated by Algorithm 1 has a subsequence $\left\{\left(\sigma_{k_{m}}^{*}, u_{k_{m}}^{*}, U_{k_{m}}^{*}, p_{k_{m}}^{*}, P_{k_{m}}^{*}\right)\right\}$ converging to a solution $\left(\sigma^{*}, u^{*}, U^{*}, p^{*}, P^{*}\right)$ to the continuous optimality system (2.5) in the following sense:

$$
\left\|\sigma_{k_{m}}^{*}-\sigma^{*}\right\|_{H^{1}(\Omega)},\left\|\left(u_{k_{m}}^{*}-u^{*}, U_{k_{m}}^{*}-U^{*}\right)\right\|_{\mathbb{H}, *},\left\|\left(p_{k_{m}}^{*}-p^{*}, P_{k_{m}}^{*}-P^{*}\right)\right\|_{\mathbb{H}, *} \rightarrow 0 \quad \text { as } m \rightarrow \infty .
$$


REMARK 4.11 Theorem 4.10 is only concerned with the convergence of the adaptive solution to the continuous Tikhonov solution, which is limited by the data accuracy (i.e., the noise level $\delta$ ) and regularization parameter $\alpha$. In the spirit of the classical discrepancy principle (Ito \& Jin (2015)), it is unnecessary to make the adaptive FEM approximation of the forward model far more accurate than the data accuracy. In practice, it is advisable to terminate the refinement step when the estimator $\eta_{k}$ falls below a multiple of the noise level $\delta$, however, the regularizing property (and the convergence rate) of such a procedure is still to be studied.

\section{Numerical experiments and discussions}

In this section, we present numerical results to illustrate the convergence and efficiency of the adaptive algorithm. All the computations were carried out using MATLAB 2013a on a personal laptop with $6.00 \mathrm{~GB}$ RAM and $2.5 \mathrm{GHz}$ CPU. The setup of the numerical experiments is as follows. The domain $\Omega$ is taken to be a square $\Omega=(-1,1)^{2}$. There are sixteen electrodes $\left\{e_{l}\right\}_{l=1}^{L}$ (with $L=16$ ) evenly distributed along the boundary $\Gamma$, each of the length $1 / 4$, thus occupying one half of the boundary $\Gamma$. The contact impedances $\left\{z_{l}\right\}_{l=1}^{L}$ on the electrodes $\left\{e_{l}\right\}_{l=1}^{L}$ are all set to unit, and the background conductivity $\sigma_{0}$ is taken to be $\sigma_{0} \equiv 1$. For each example, we measure the electrode voltages $U$ for the first ten sinusoidal input currents, in order to gain enough information about the true conductivity $\sigma^{\dagger}$. Then the noisy data $U^{\delta}$ is generated by adding componentwise Gaussian noise to the exact data $U\left(\sigma^{\dagger}\right)$ as follows

$$
U_{l}^{\delta}=U_{l}\left(\sigma^{\dagger}\right)+\varepsilon \max _{l}\left|U_{l}\left(\sigma^{\dagger}\right)\right| \xi_{l}, \quad l=1, \ldots, L,
$$

where $\varepsilon$ is the (relative) noise level, and $\left\{\xi_{l}\right\}$ follow the standard normal distribution. The exact data $U\left(\sigma^{\dagger}\right)$ is computed on a much finer mesh generated adaptively (and thus completely different from the one used in the inversion), in order to avoid the most obvious form of "inverse crime". In all the experiments, the marking strategy (3.6) in the module MARK is represented by a specific maximum strategy, cf. Remark 3.2, i.e., mark a minimal subset $\mathscr{M}_{k} \subseteq \mathscr{T}_{k}$, i.e., the refinement set, such that

$$
\eta_{k}\left(\sigma_{k}^{*}, u_{k}^{*}, U_{k}^{*}, p_{k}^{*}, P_{k}^{*}, \mathscr{M}_{k}\right) \geqslant \theta \eta_{k}\left(\sigma_{k}^{*}, u_{k}^{*}, U_{k}^{*}, p_{k}^{*}, P_{k}^{*}, \mathscr{T}_{k}\right),
$$

with a threshold $\theta \in(0,1]$. In the computation, we fix the threshold $\theta$ at $\theta=0.7$. For the adaptive refinement, we employ the newest vertex bisection to subdivide the marked triangles; see Mitchell (1989) for implementation details. The discrete nonlinear optimization problems (3.1)-(3.2) are solved by a nonlinear conjugated gradient method, where the box constraints are enforced by pointwise projection into the admissible set $\mathscr{A}$ after each update, and the initial guess of the conductivity at the coarsest mesh $\mathscr{T}_{0}$ is initialized to the background conductivity $\sigma_{0}=1$, and then for $k=1,2, \ldots$, the reconstruction on the mesh $\mathscr{T}_{k-1}$ is interpolated to the mesh $\mathscr{T}_{k}$ to warm start the (projected) conjugate gradient iteration for the discrete optimization problem on the mesh $\mathscr{T}_{k}$. Throughout the adaptive loop, the regularization parameter $\alpha$ in the model (2.3) is fixed and it is determined in a trial-and-error manner, and the chosen values of $\alpha$ in the experiments below are roughly of the order of the noise level $\delta$, which is a popular a priori parameter choice; see Ito \& Jin (2015) for further discussions about parameter choice. It is an interesting research question to adapt the choice of $\alpha$ with the a posterior estimator $\eta_{k}$ within the adaptive algorithm; see Remark 4.11.

EXAMPLE 5.1 The true conductivity $\sigma^{\dagger}$ is given by $\sigma^{\dagger}(x)=\sigma_{0}(x)+e^{-8\left(x_{1}^{2}+\left(x_{2}-0.55\right)^{2}\right)}$, with the background conductivity $\sigma_{0}(x)=1$.

In this example, the true conductivity $\sigma^{\dagger}$ consists of a very smooth blob in a constant background, and the profile is shown in Fig. 1(a). The final recovered conductivity fields from the voltage measurements with $\varepsilon=0.1 \%$ data noise are shown in Fig. 1. For both uniform and adaptive refinements, the recoveries capture 
well the location and height of the blob: it is very smooth, due to the use of a smoothness prior. Hence, it does not induce any grave solution singularity. The recoveries by both methods are similar to each other in terms of location and magnitude. Both suffer from a slight loss of the contrast, which is typical for EIT recoveries with a smoothness penalty; see, e.g., Lechleiter \& Rieder (2006) and Winkler \& Rieder (2014) for similar results by an iteratively regularized Gauss-Newton method.

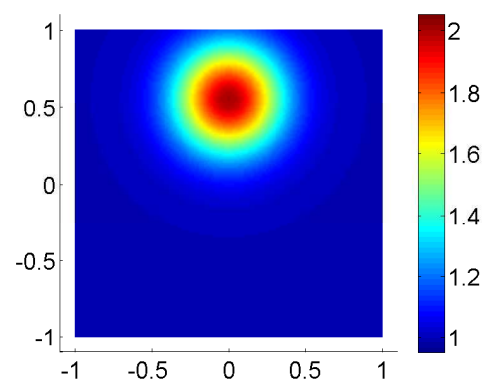

(a) true conductivity

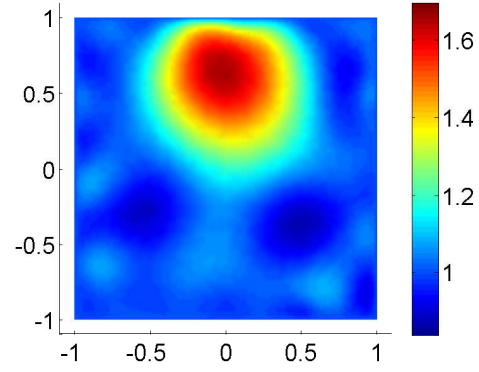

(b) adaptive refinement

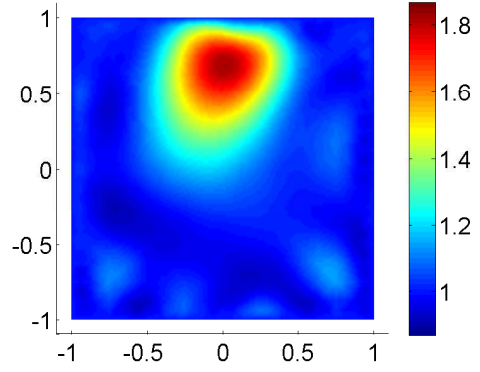

(c) uniform refinement

FIG. 1. The final reconstructions by the uniform and adaptive refinements for Example 5.1 with $\varepsilon=0.1 \%$ noise in the data. The degree of freedom is 9818 and 16641 for the adaptive and uniform refinement, respectively. The regularization parameter $\alpha$ is fixed at $\alpha=2.5 \times 10^{-4}$

Next we examine the adaptive refinement more closely. On a very coarse initial mesh $\mathscr{T}_{0}$, which is a uniform triangulation of the domain $\Omega$, cf. Fig. 2(a), the recovered conductivity tends to have pronounced oscillations around the boundary, since the forward solution is not accurately resolved over there. In particular, the discretization error significantly compromises the reconstruction accuracy, and it induces large errors in the location and height of the recovered conductivity. This motivates the use of the adaptive strategy. The meshes during the adaptive iteration and the corresponding recoveries are shown in Fig. 2. The refinement step first concentrates only on the region around the electrode surface. This is attributed to the change of the boundary condition, which induces weak singularities in the direct and adjoint solutions. Then the AFEM starts to refine also the interior of the domain, simultaneously with the boundary region. Accordingly, the spurious oscillations in the recovery are suppressed as the iteration proceeds (provided that the regularization parameter $\alpha$ is properly chosen). Interestingly, the central part of the domain $\Omega$ is refined only slightly during the whole refinement procedure, and in the end, much coarse elements are used for the conductivity inversion in these regions. This concurs with the empirical observation that the inclusion in the central part is much harder to resolve from the boundary data. Hence, the adaptive algorithm tends to adapt automatically to the resolving power of the conductivity (from the boundary data) in different regions.

In Fig. 3, we plot the $L^{2}(\Omega)$ and $H^{1}(\Omega)$ errors of the recoveries versus the degree of freedom $N$ of the mesh $\mathscr{T}_{k}$ for the adaptive and uniform refinement, where the recovery on the finest mesh is taken as a respective reference solution, since the recoveries by the uniform and adaptive refinements are not necessarily the same (although always close), even initialized identically. The corresponding empirical convergence rates in $L^{2}(\Omega)$-norms and $H^{1}(\Omega)$-norms are given in Table 1. It is observed that with the same degree of freedom, the AFEM can give much more accurate results than the uniform one (with respect to the respective reference solution). This is also confirmed by the computing time: for the results in Fig. 1, the one by the adaptive refinement takes about 30 minutes, whereas that by the uniform refinement takes about 80 minutes. This is consistent with the fact that at each iteration of the algorithm, the module SOLVE is predominant, and that the computational cost of the conjugate gradient descent algorithm is proportional to the number of 
forward and adjoint solves at each iteration and each forward/adjoint solve is determined by the degree of freedom of the system. This shows clearly the computational efficiency of the proposed adaptive algorithm.
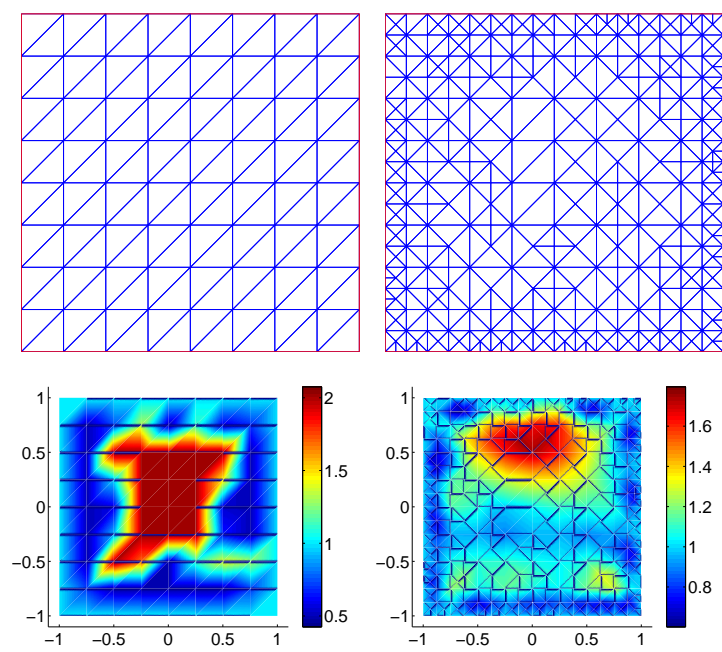

(a) 0th step

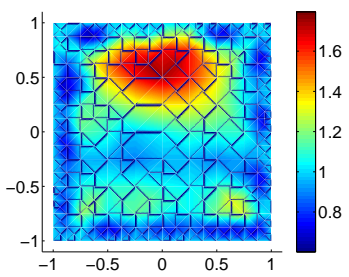

(b) 4th step
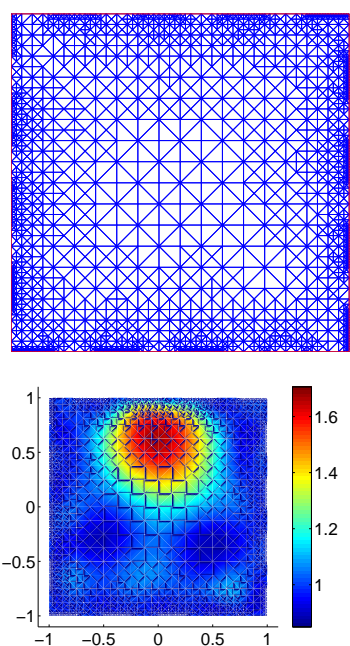

(c) 9 th step
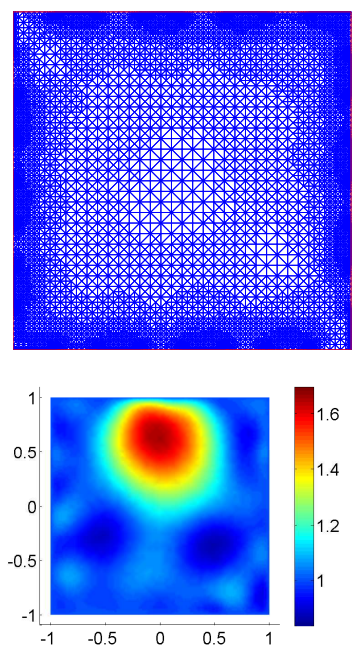

(d) 14 th step

FIG. 2. The recovered conductivity distributions $\sigma_{k}^{*}$ during the adaptive refinement, for Example 5.1 with $\varepsilon=0.1 \%$ noise. The regularization parameter $\alpha$ is fixed at $\alpha=2.5 \times 10^{-4}$.

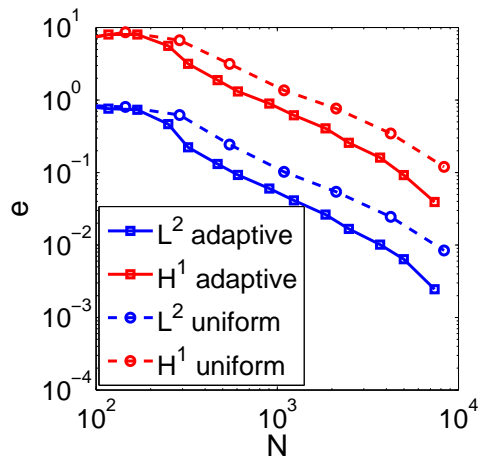

(a) $\varepsilon=1 \times 10^{-3}$

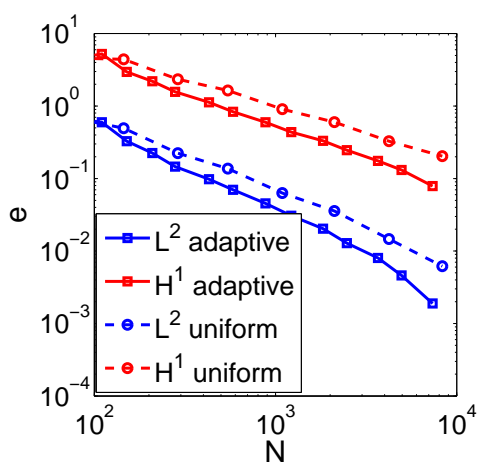

(b) $\varepsilon=1 \times 10^{-2}$

FIG. 3. The $L^{2}(\Omega)$ and $H^{1}(\Omega)$ errors versus the degree of freedom $N$ of the mesh, for Example 5.1 at two different noise levels, using the adaptive refinement (solid line) and uniform refinement (dashed line).

A second example contains two neighboring smooth blobs.

EXAMPLE 5.2 The true conductivity $\sigma^{\dagger}$ is given by $\sigma^{\dagger}(x)=\sigma_{0}(x)+e^{-20\left(\left(x_{1}+0.7\right)^{2}+x_{2}^{2}\right)}+e^{-20\left(x_{1}^{2}+\left(x_{2}-0.7\right)^{2}\right)}$, and the background conductivity $\sigma_{0}(x)=1$.

Like before, the true conductivity $\sigma^{\dagger}$ is smooth (cf. Fig. 4(a) for the profile), and thus the smoothness 
Table 1. The empirical convergence rates $O\left(N^{-r}\right), N$ being the degree of freedom, of the recoveries in the $L^{2}(\Omega)$ - and $H^{1}(\Omega)$-norms, for the numerical examples, where the exponent $r$ is presented.

\begin{tabular}{|c|cc|cc|cc|cc|}
\hline \multirow{3}{*}{ Example } & \multicolumn{4}{|c|}{$\varepsilon=1 \mathrm{e}-3$} & \multicolumn{4}{c|}{$\varepsilon=1 \mathrm{e}-2$} \\
\cline { 2 - 9 } & \multicolumn{2}{|c|}{ adaptive } & \multicolumn{2}{|c|}{ uniform } & \multicolumn{2}{c|}{ adaptive } & \multicolumn{2}{c|}{ uniform } \\
\cline { 2 - 9 } & $L^{2}$ & $H^{1}$ & $L^{2}$ & $H^{1}$ & $L^{2}$ & $H^{1}$ & $L^{2}$ & $H^{1}$ \\
\hline 5.1 & 1.31 & 1.19 & 1.04 & 0.93 & 1.23 & 0.91 & 1.01 & 0.70 \\
5.2 & 1.32 & 1.19 & 1.05 & 0.94 & 1.23 & 0.88 & 0.99 & 0.73 \\
5.3 & 1.08 & 0.88 & 0.83 & 0.73 & 0.91 & 0.67 & 0.72 & 0.40 \\
\hline
\end{tabular}

penalty is suitable. Overall, the observations from Example 5.1 remain valid: the recovered coefficient captures very well the supports of the inclusions, and the magnitude is also reasonable. The recovery by the adaptive algorithm is comparable with that based on uniform one, but requiring far less degrees of freedom. However, due to the smoothing nature of the $H^{1}(\Omega)$ penalty, the recoveries tend to be diffusive, and the magnitude also suffers from a loss of about $20 \%$ for both uniform and adaptive refinements. Such smoothing is well-known in EIT imaging. These drawbacks can be partially alleviated by sparsity-promoting penalty (Jin et al. (2012), Jin \& Maass (2012b)), to which it is of great interest to extend the proposed AFEM.

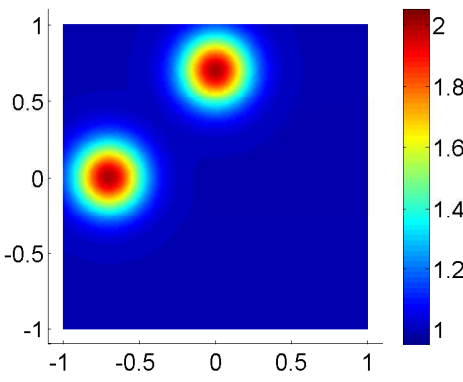

(a) true conductivity

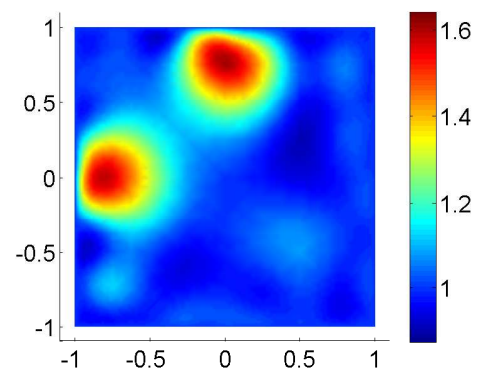

(b) adaptive refinement

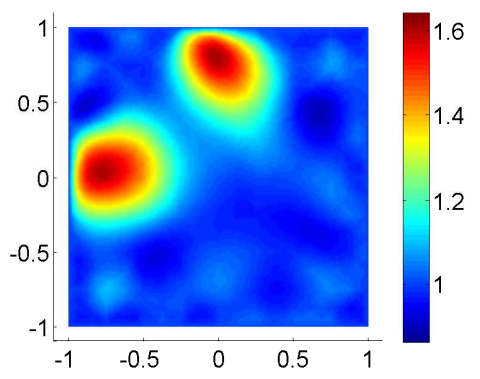

(c) uniform refinement

FIG. 4. The final reveries by the adaptive and uniform refinements for Example 5.2 with $\varepsilon=0.1 \%$ noise. The degree of freedom is 9803 and 16641 for the adaptive and uniform refinement, respectively. The regularization parameter $\alpha$ is fixed at $\alpha=2.5 \times 10^{-4}$.

We plot in Fig. 5 the meshes and recoveries at the intermediate refinement steps. At the initial stage, the refinement mainly occurs in the region around electrode surfaces, where the weak solution singularity appears. As the refinement proceeds, the region away from the boundary is also refined, but to a much lesser degree, especially for the central part of the domain. In case of a very coarse initial mesh, the recovery even fails to correctly identify the number of inclusions, but as the AFEM proceeds, the spurious oscillations disappear, and then it can identify reasonably the locations and magnitudes of the blobs from the recoveries, cf. Fig. 5. In Fig. 6, we show the $L^{2}(\Omega)$ and $H^{1}(\Omega)$ errors of the recoveries versus the degree of freedom $N$ of the mesh $\mathscr{T}_{k}$ for the adaptive and uniform refinement. These plots fully show the efficiency of the adaptive algorithm, for both $\varepsilon=0.1 \%$ and $\varepsilon=1 \%$ noise; see also Table 1 for the empirical convergence rates.

Last, we consider one example with a discontinuous conductivity field. 

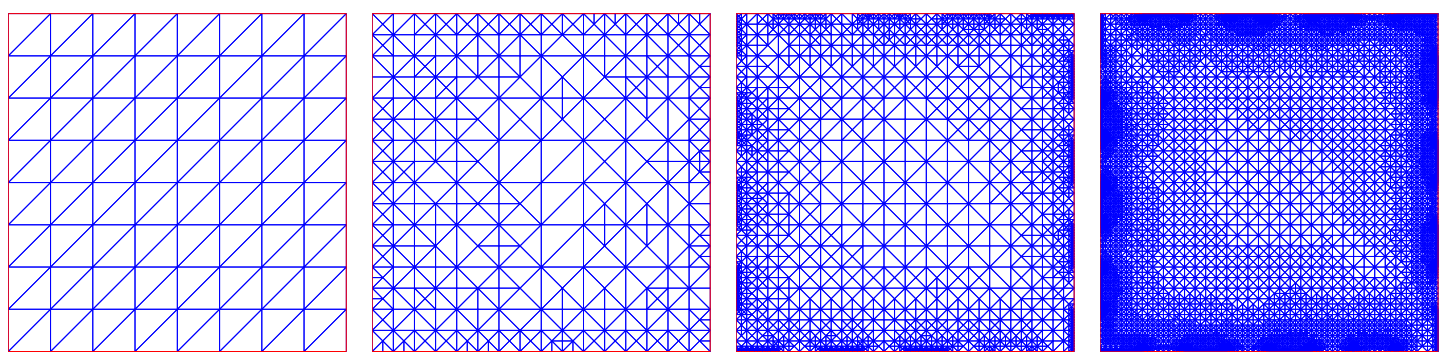

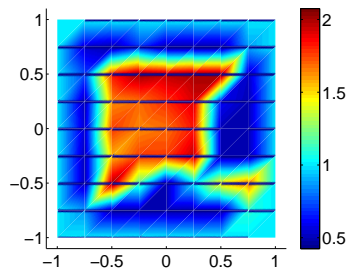

(a) 0th step

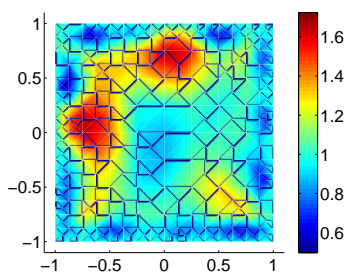

(b) 4th step

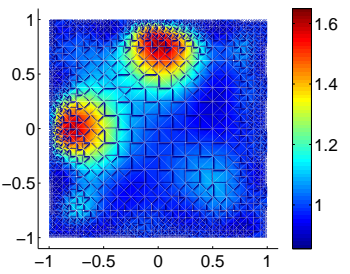

(c) 9 th step

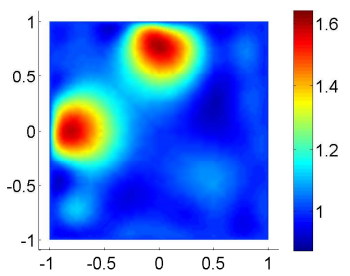

(d) 14th step

FIG. 5. The recovered conductivity during the adaptive refinement, for Example 5.2 with $\varepsilon=0.1 \%$ noise. The regularization parameter is fixed at $\alpha=2.5 \times 10^{-4}$.

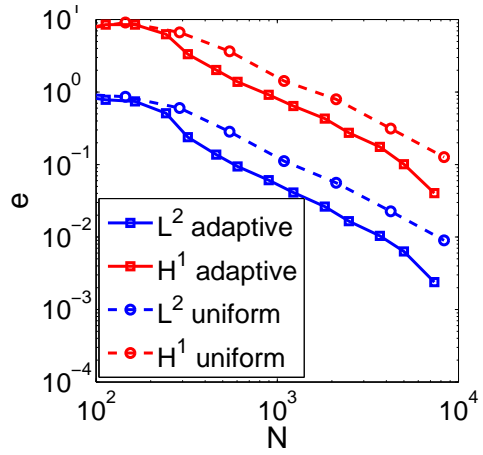

(a) $\varepsilon=1 \times 10^{-3}$

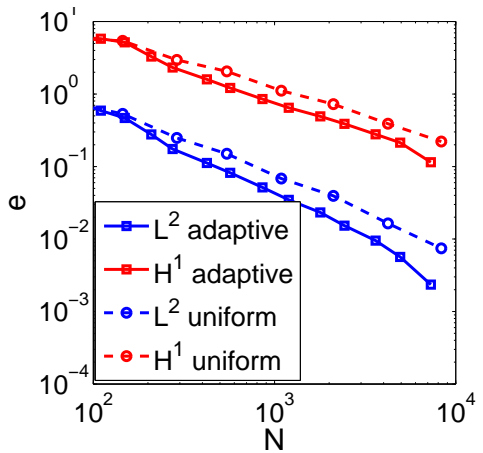

(b) $\varepsilon=1 \times 10^{-2}$

FIG. 6. The $L^{2}(\Omega)$ and $H^{1}(\Omega)$ errors versus the degree of freedom $N$ of the mesh, for Example 5.2 at two different noise levels, using the adaptive refinement (solid line) and uniform refinement (dashed line).

EXAMPLE 5.3 The true conductivity $\sigma^{\dagger}$ is given by $\sigma^{\dagger}(x)=\sigma_{0}(x)+\left(x_{1} / 2+x_{2}\right) \chi_{\Omega^{\prime}}$, where $\chi_{\Omega^{\prime}}$ is the characteristic function of the set $\Omega^{\prime}=(1 / 4,3 / 4) \times(0,1 / 2)$, and the back ground conductivity $\sigma_{0}(x)=1$.

Since the $H^{1}(\Omega)$ penalty imposes a global smoothness condition, it is unsuitable for recovering discontinuous conductivity fields. Hence, in this example we assume that the support $\Omega^{\prime}$ of the true conductivity field $\sigma^{\dagger}$ is known, and aim at determining the variation within the support using the $H^{1}\left(\Omega^{\prime}\right)$ semi-norm penalty. The adaptive algorithm and the convergence proof can be extended directly: the variational inequality is now defined only on $\Omega^{\prime}$, and the estimator $\eta_{\mathscr{T}, 3}^{2}\left(\sigma_{\mathscr{T}}^{*}, u_{\mathscr{T}}^{*}, p_{\mathscr{T}}^{*}, T\right)$ is only for elements in $\left.\Omega^{\prime}\right)$.

The numerical results for the example are presented in Figs. 7, 8 and 9. The observations from the preceding two examples remain largely valid. The magnitude of the conductivity is slightly reduced, but otherwise the profile is reasonable, and visually the recoveries by the adaptive and the uniform refinements 
are close to each other, cf. Figs. 7(b) and 7(c). Even though the conductivity field $\sigma$ is discontinuous, the adaptive algorithm first mainly resolves the singularity due to the change of boundary conditions, i.e., around the boundary, cf. Fig. 8(b). As the adaptive iteration proceeds, the algorithm then starts to refine the region near the boundary $\partial \Omega^{\prime}$ of the subdomain $\Omega^{\prime}$ : first the part close to the boundary $\partial \Omega$, and then the part away from $\partial \Omega$, cf. Figs. 8(c) and 8(d). This is consistent with the empirical observation that the further away from the boundary, the more challenging it is to be resolved (from the boundary data), i.e., the boundary data allows better resolving the regions close to the boundary. Hence, the solution singularity induced by the conductivity discontinuity does not play an important role in the inversion as it was in direct problems. The gain of computational efficiency is shown in Fig. 9: the $L^{2}(\Omega)$ - and the $H^{1}(\Omega)$-errors decrease faster with the increase of degree of freedom for the adaptive algorithm than that for the uniform refinement.

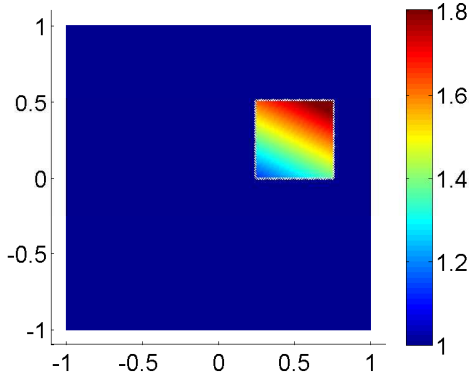

(a) true conductivity

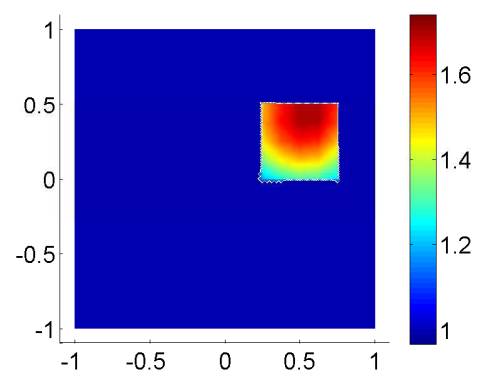

(b) adaptive refinement

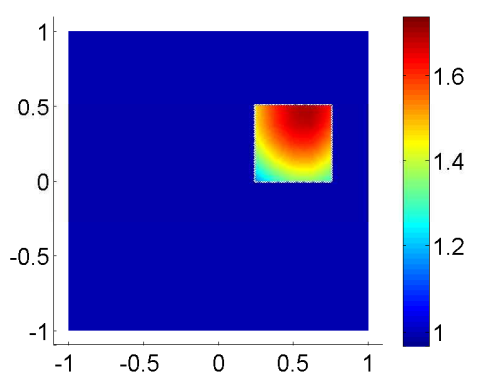

(c) uniform refinement

FIG. 7. The final reveries by the adaptive and uniform refinements for Example 5.3 with $\varepsilon=0.1 \%$ noise. The degree of freedom is 19608 and 33025 for the adaptive and uniform refinement, respectively. The regularization parameter $\alpha$ is fixed at $\alpha=3.2 \times 10^{-3}$.

\section{Concluding remarks}

In this work, we have developed a novel adaptive finite element method for the electrical impedance tomography inverse problem, modeled by the complete electrode model. It is formulated as an output leastsquares problem with a Sobolev smoothness penalty. The weak solution singularity around the electrode surfaces and low-regularity conductivity motivate the use of the adaptive refinement techniques. We have derived a residual-type a posteriori error estimator, which involves the state, adjoint and conductivity estimate, and established the convergence of the sequence of solutions generated by the adaptive technique that the accumulation point solves the continuous optimality system. The efficiency and convergence of the proposed adaptive algorithm is confirmed by a few numerical experiments.

This work represents only a first step towards the rigorous adaptive finite element method for nonlinear inverse problems associated with PDEs. There are several research problems deserving further study. First, the proposed algorithm is only for the smoothness penalty, which is essential in the development and convergence analysis of the algorithm. It is of much interest to derive and to analyze adaptive algorithms for nonsmooth penalties, e.g., total variation and sparsity. Second, numerically one observes that the algorithm can approximate a (local/global) minimizer of the continuous optimization well, instead of only a solution to the necessary optimality condition. This is still theoretically to be justified. Third, the reliability and optimality of the adaptive algorithm for nonlinear inverse problems are completely open, which seems not fully 

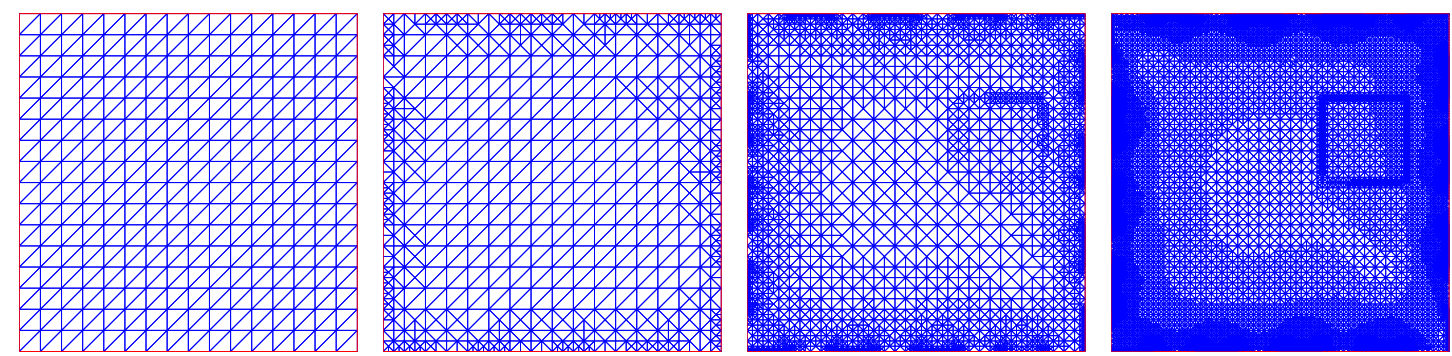

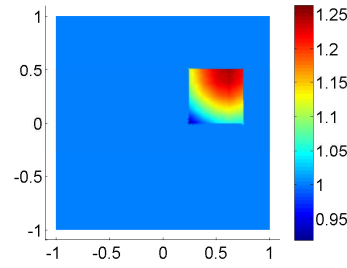

(a) 0th step

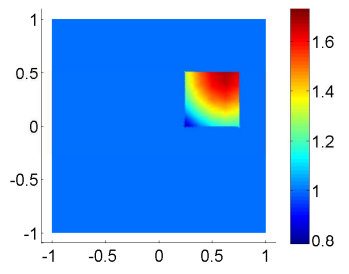

(b) 4th step

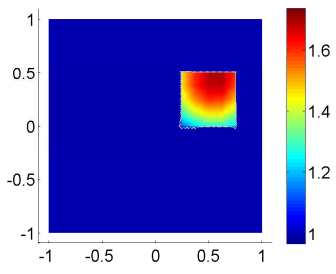

(c) 9 th step

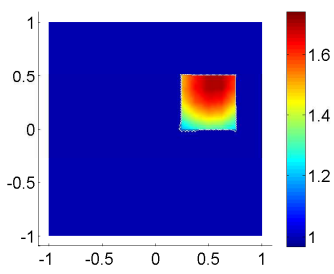

(d) 14th step

FIG. 8. The recovered conductivity during the adaptive refinement, for Example 5.3 with $\varepsilon=0.1 \%$ noise. The regularization parameter is fixed at $\alpha=3.2 \times 10^{-3}$.

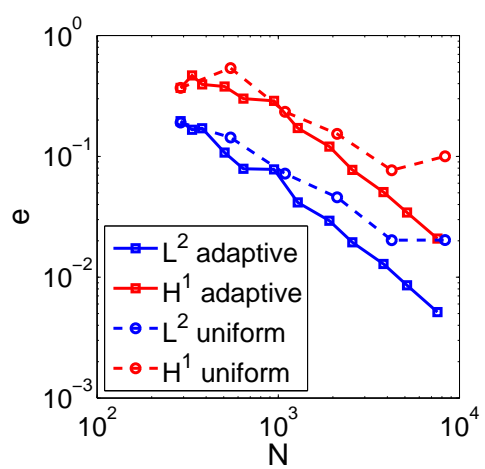

(a) $\varepsilon=1 \times 10^{-3}$

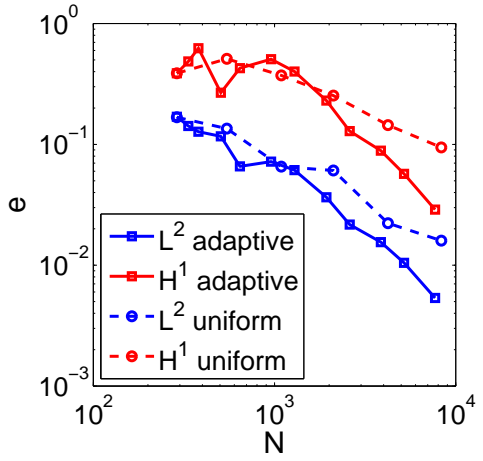

(b) $\varepsilon=1 \times 10^{-2}$

FIG. 9. The $L^{2}\left(\Omega^{\prime}\right)$ and $H^{1}\left(\Omega^{\prime}\right)$ errors versus the degree of freedom $N$ of the mesh, for Example 5.3 at two different noise levels, using the adaptive refinement (solid line) and uniform refinement (dashed line).

understood even for linear ones. The optimality issue in the context of inverse problems should be related to the noise level. The crucial interplay between the error estimator and noise level is to be elucidated.

\section{Acknowledgements}

The authors are grateful to the referees for their constructive comments, which have led to an improved presentation, and to Mr. Chun-Man Yuen for his great help in carrying out the numerical experiments. 


\section{Funding}

The work of B. Jin was supported by UK EPSRC grant EP/M025160/1, and that of Y. Xu by National Natural Science Foundation of China (11201307), Ministry of Education of China through Specialized Research Fund for the Doctoral Program of Higher Education (20123127120001), E-Institute of Shanghai Universities (E03004) and Innovation Program of Shanghai Municipal Education Commission (13YZ059). The work of J. Zou was substantially supported by Hong Kong RGC grants (projects 14306814 and 405513).

\section{REFERENCES}

Adler, A., Gaburro, R. \& Lionheart, W. (2011) Electrical impedance tomography. Handbook of Mathematical Methods in Imaging (O. Scherzer ed.). Springer-Verlag.

Ainsworth, M. \& Oden, J. T. (2000) A Posteriori Error Estimation in Finite Element Analysis. New York: WileyInterscience.

BECKeR, R. \& MAO, S. (2011) Quasi-optimality of an adaptive finite element method for an optimal control problem. Comput. Methods Appl. Math., 11, 107-128.

BECKER, R. \& VEXLER, B. (2004) A posteriori error estimation for finite element discretization of parameter identification problems. Numer. Math., 96, 435-459.

Beilina, L., Klibanov, M. V. \& Kokurin, M. Y. (2010) Adaptivity with relaxation for ill-posed problems and global convergence for a coefficient inverse problem. J. Math. Sci., 167, 279-325.

BEILINA, L. \& CLASON, C. (2006) An adaptive hybrid FEM/FDM method for an inverse scattering problem in scanning acoustic microscopy. SIAM J. Sci. Comput., 28, 382-402.

Beilina, L. \& Johnson, C. (2005) A posteriori error estimation in computational inverse scattering. Math. Models Methods Appl. Sci., 15, 23-35.

Beilina, L. \& Klibanov, M. V. (2010a) A posteriori error estimates for the adaptivity technique for the tikhonov functional and global convergence for a coefficient inverse problem. Inverse Problems, 26, 045012, 27pp.

Beilina, L. \& Klibanov, M. V. (2010b) Reconstruction of dielectrics from experimental data via a hybrid globally convergent/adaptive algorithm. Inverse Problems, 26, 125009, $30 \mathrm{pp}$.

Carstensen, C., Feischl, M., Page, M. \& Praetorius, D. (2014) Axioms of adaptivity. Comput. Math. Appl., 67, 1195-1253.

Cheng, K.-S., IsAacson, D., Newell, J. C. \& Gisser, D. G. (1989) Electrode models for electric current computed tomography. IEEE Trans. Biomed. Eng., 36, 918-924.

Chow, Y. T., Iто, K. \& Zou, J. (2014) A direct sampling method for electrical impedance tomography. Inverse Problems, 30, 095003, 25 pp.

Ciarlet, P. G. (2002) The Finite Element Method for Elliptic Problems. Philadelphia, PA: SIAM.

Dunlop, M. M. \& StUART, A. M. (2015). The Bayesian formulation of EIT: analysis and algorithms. preprint, arXiv:1508.04106.

Evans, L. C. \& GARIEPy, R. F. (1992) Measure Theory and Fine Properties of Functions. Boca Raton: CRC Press, pp. viii +268 .

FenG, T., YAn, Y. \& LiU, W. (2008) Adaptive finite element methods for the identification of distributed parameters in elliptic equation. Adv. Comput. Math., 29, 27-53.

GeHre, M., Jin, B. \& LU, X. (2014) An analysis of finite element approximation of electrical impedance tomography. Inverse Problems, 30, 045013, 24 pp.

GEHRE, M. \& JIN, B. (2014) Expectation propagation for nonlinear inverse problems with an application to electrical impedance tomography. J. Comput. Phys., 259, 513-535.

Griesbaum, A., Kaltenbacher, B. \& Vexler, B. (2008) Efficient computation of the Tikhonov regularization parameter by goal-oriented adaptive discretization. Inverse Problems, 24, 025025, 20.

Grisvard, P. (1985) Elliptic Problems in Nonsmooth Domains. Pitman, Boston, MA, pp. xiv+410. 
HARRACH, B. \& UlLRich, M. (2013) Monotonicity-based shape reconstruction in electrical impedance tomography. SIAM J. Math. Anal., 45, 3382-3403.

Hintermüller, M., Hoppe, R. H. W., Iliash, Y. \& Kieweg, M. (2008) An a posteriori error analysis of adaptive finite element methods for distributed elliptic control problems with control constraints. ESAIM, Control Optim. Calc. Var., 14, 540-560.

Hintermüller, M. \& Hoppe, R. H. W. (2010) Goal-oriented adaptivity in pointwise state constrained optimal control of partial differential equations. SIAM J. Control Optim., 48, 5468-5487.

ITO, K. \& Jin, B. (2015) Inverse Problems: Tikhonov Theory and Algorithms. Series on Applied Mathematics, vol. 22. World Scientific Publishing Co. Pte. Ltd., Hackensack, NJ, pp. x+318.

ITo, K. \& Kunisch, K. (2008) Lagrange Multiplier Approach to Variational Problems and Applications. SIAM, Philadelphia, PA, pp. xviii+341.

Jin, B., KhAN, T. \& MAASs, P. (2012) A reconstruction algorithm for electrical impedance tomography based on sparsity regularization. Internat. J. Numer. Methods Engrg., 89, 337-353.

JIN, B. \& MAASS, P. (2012a) An analysis of electrical impedance tomography with applications to Tikhonov regularization. ESAIM: Control, Optim. Calc. Var., 18, 1027-1048.

Jin, B. \& MAASS, P. (2012b) Sparsity regularization for parameter identification problems. Inverse Problems, 28, $123001,70 \mathrm{pp}$

Kaltenbacher, B., Kirchner, A. \& Veljović, S. (2014) Goal oriented adaptivity in the IRGNM for parameter identification in PDEs: I. reduced formulation. Inverse Problems, 30, 0450011, 26.

Knudsen, K., Lassas, M., Mueller, J. L. \& Siltanen, S. (2009) Regularized D-bar method for the inverse conductivity problem. Inverse Probl. Imaging, 3, 599-624.

KossaCZKÝ, I. (1995) A recursive approach to local mesh refinement in two and three dimensions. J. Comput. Appl. Math., 55, 275-288.

Lechleiter, A., Hyvönen, N. \& Hakula, H. (2008) The factorization method applied to the complete electrode model of impedance tomography. SIAM J. Appl. Math., 68, 1097-1121.

LEChleiter, A. \& RiEder, A. (2006) Newton regularizations for impedance tomography: a numerical study. Inverse Problems, 22, 1967-1987.

LI, J., XIE, J. \& Zou, J. (2011) An adaptive finite element reconstruction of distributed fluxes. Inverse Problems, 27, 075009, 25pp.

LI, R., LiU, W., MA, H. \& TANG, T. (2002) Adaptive finite element approximation for distributed elliptic optimal control problems. SIAM J. Control Optim., 41, 1321-1349.

LIU, W. \& YAN, N. (2001) A posteriori error estimates for distributed convex optimal control problems. Adv. Comput. Math., 15, 285-309.

Mitchell, W. F. (1989) A comparison of adaptive refinement techniques for elliptic problems. ACM Trans. Math. Software, 15, 326-347 (1990).

Nochetto, R. H., Siebert, K. G. \& Veeser, A. (2009) Theory of adaptive finite element methods: an introduction. Multiscale, Nonlinear and Adaptive Approximation (R. A. DeVore \& A. Kunoth eds). New York: Springer, pp. 409-542.

SCOTT, L. R. \& ZHANG, S. (1990) Finite element interpolation of nonsmooth functions satisfying boundary conditions. Math. Comp., 54, 483-493.

SiEberT, K. G. (2011) A convergence proof for adaptive finite elements without lower bounds. IMA J. Num. Anal., 31, 947-970.

Somersalo, E., Cheney, M. \& IsaAcson, D. (1992) Existence and uniqueness for electrode models for electric current computed tomography. SIAM J. Appl. Math., 52, 1023-1040.

Verfürth, R. (1996) A Review of A Posteriori Estimation and Adaptive Mesh-Refinement Techniques. Chichester, New York, Stuttgart: Wiley-Teubner.

WINKLER, R. \& RIEDER, A. (2014) Resolution-controlled conductivity discretization in electrical impedance tomography. SIAM J. Imaging Sci., 7, 2048-2077. 
XU, Y. \& Zou, J. (2015a) Analysis of an adaptive finite element method for recovering the Robin coefficient. SIAM J. Control Optim., 53, 622-644.

XU, Y. \& ZoU, J. (2015b) Convergence of an adaptive finite element method for distributed flux reconstruction. Math. Comp., 84, 2645-2663. 